\title{
Dynamic Model and Control of a New Underwater Three-Degree-of-Freedom Tidal Energy Converter
}

\author{
José A. Somolinos, ${ }^{1}$ Amable López, ${ }^{1}$ Marina P. Portilla, ${ }^{1}$ and Rafael Morales ${ }^{2}$ \\ ${ }^{1}$ Grupo de Investigación Tecnológico en Energías Renovables Marinas (GIT-ERM), E.T.S. de Ingenieros Navales, \\ Universidad Politécnica de Madrid, Arco de la Victoria 4, 28040 Madrid, Spain \\ ${ }^{2}$ E.T.S. de Ingenieros Industriales, Universidad de Castilla-La Mancha, Campus Universitario, s/n, 02071 Albacete, Spain
}

Correspondence should be addressed to Rafael Morales; rafael.morales@uclm.es

Received 6 May 2015; Accepted 28 June 2015

Academic Editor: Sergey A. Suslov

Copyright (C) 2015 José A. Somolinos et al. This is an open access article distributed under the Creative Commons Attribution License, which permits unrestricted use, distribution, and reproduction in any medium, provided the original work is properly cited.

\begin{abstract}
There is currently a growing interest in developing devices that can be used to exploit energy from oceans. In the recent past, the search for oil and gas at ever-greater depths has led to the evolution of devices with which these resources are extracted. These devices range from those that simply rest on the seabed to those that are fully floating and anchored to it. This trend can be considered as the basis needed to understand the future evolution of devices for harnessing depth renewable resources. This paper presents a simple dynamic modeling and a nonlinear multivariable control model-based system for a new three-degree-of-freedom underwater generator with which energy from depth marine currents is harnessed when reference trajectory tracking for the emersion maneuvers needed to carry out maintenance tasks is performed. The goodness of both the model and the proposed controller has been demonstrated through the development of various simulations in the MATLAB-Simulink environment. Additionally, the validation of the control algorithms was carried out by using the dynamic model offered by the simulation environment Orcina OrcaFlex (software for the dynamic analysis for offshore marine systems) through the MATLAB-OrcaFlex interface.
\end{abstract}

\section{Introduction}

The growing interest in the exploitation of marine renewable energies began several years ago, and various devices with which to harness energy from seas have therefore been conceived or developed (see [1-3]), their main natural energy sources being wind, waves, and marine currents.

One of the most promising sources of marine energy is the exploitation of tidal or oceanic water flows [4-7], and the industry's effort is currently focused on the so-called first generation devices [8] (fixed to the sea bottom and suitable for sites with depths below $40 \mathrm{~m}$ ). But there are an increasing number of second-generation devices that have been conceived to be moored to the sea bottom with an expected similar trend to that which has taken place during the development of oil and gas platforms that must access resources at increasingly greater depths [9].

This evolution has led these devices to evolve from being anchored to the seabed to being located in a floating location, and the most appropriate ways and means to perform maintenance tasks have therefore also had to evolve these kinds of devices [10-15].

The successful installation of these kinds of devices for harnessing energy from depth currents can only take place once it has been proved that they are both technically and economically feasible in comparison to other traditional energy sources. One well known way in which to reduce costs is by successively automating more tasks, thus signifying less human intervention or the possibility of using the cheapest general purpose ships rather than high cost special vessels for maintenance purposes. In [16] are studied the automatic maneuvering emersion and immersion of one of these devices.

Nowadays there exist different devices with the following main alternatives for performing maintenance tasks:

(i) There is the use of a servo actuated crabbing based system to move the main generation unit from the support structure. See different devices from $[17,18]$. 
(ii) There is the use of elevation and placement by means of floating cranes. See [19-23].

(iii) There is the use of a ballast management system to generate vertical forces, thus enabling the device's emersion and immersion movements to be controlled. See [24-26].

A new family of generators is briefly presented in this work. They use a mooring system based on buoys and wires that allow the device to be located at the desired position on the seabed and positioned at any desired depth of the layer with almost no human resources. By simply disconnecting stern wire that joins the generator to the seabed and a proper management of the ballast water that is strategically located inside the generator, it is possible to perform closed loop emersion and immersion maneuvers with three degrees of freedom (DoFs), two orientation angles and a depth control, in such a way that the process of moving from a submerged and vertically disposed state to a floating and horizontally disposed one and vice versa can be carried out fully automatically without any kind of human intervention.

This paper presents a new dynamic model for this new family of second-generation tidal energy converters $[8,27$, $28]$ for simulation and control design purposes [29, 30]. The proposed model is very simple, fully parameterized, and easily scalable with minimum computer effort. Very simple models have already been used to control complex devices with a good performance and good correspondence among simulated and experimental signals. For example, in [31], a simple model based on only one lumped mass and a special kinematic uncoupling design was used to model a threedegree-of-freedom flexible arm with complex nonlinear and time variant dynamics. This simplified dynamic model was employed as a basis for various controllers, which were used to control this robot with excellent results [32]. Another different system, in this case, a stair-climbing mobility system, was also modeled with only one lumped mass [33] and this simple dynamic model has been used as the basis for various control systems (see, a.e., [34]).

In a similar manner to that shown above, the dynamic model proposed in this paper is based on the computation of the values of only four lumped masses, which are handled solely by buoying forces applied to three star-shaped distributed equilateral torpedoes on the pod (nacelle) of the generator. The model developed exhibits time-varying, nonlinear, and strongly coupled behavior. It was, meanwhile, necessary to design the control law based on the dynamic model developed in such a way that it would have a successful closed loop behavior when the underwater three-DoF tidal energy converter performs emersion and immersion maneuvers with only passive buoyancy forces. In this work, the proposed control system is characterized by its simplicity, computational efficiency, and easy implementation in a microprocessor-/microcontroller-based system. The proposed control law is composed of the following two main terms: (i) a matrix that is responsible for decoupling the open loop dynamics from one degree of freedom with regard to the others and (ii) a feedback controller based on centrifugal

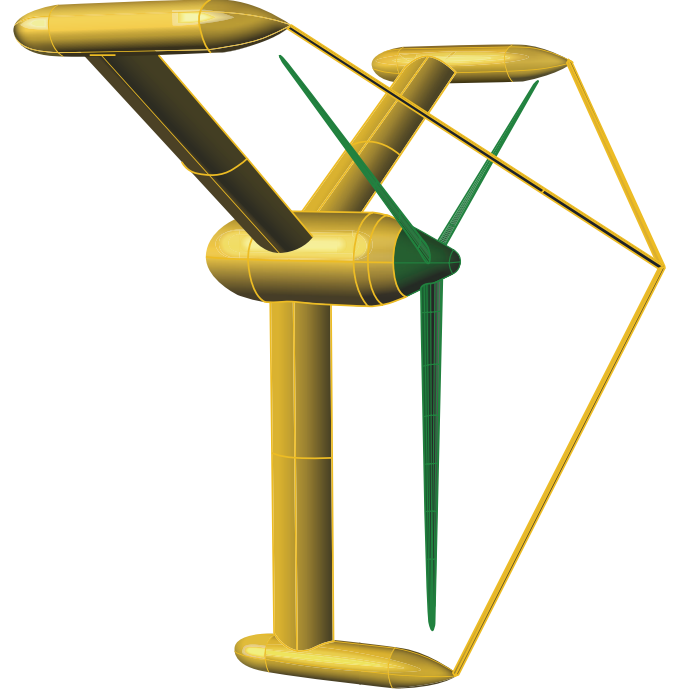

FIGURE 1: Main appearance of a generator for unidirectional currents.

and Coriolis force cancellation and a simple multivariable and diagonal PD controller.

The paper is organized as follows: after providing a brief description of the proposed family of generators in Section 2, Section 3 shows how the derivation of the proposed dynamic model of the submerged device was derived when performing coupled three-degree-of-freedom motions. Section 4 proposes the solution adopted to control the system. Section 5 presents the numerical simulations obtained to validate the proposed dynamic model and the proposed control algorithm. In particular, different smooth robotic-based trajectories were used to perform uncoupled simultaneous multiple degree-of-freedom motions. Finally, Section 6 is devoted to the conclusions of the paper, along with proposals for immediate future works.

\section{System Description}

A brief description of the proposed family of generators is now presented (see [27], a.e.). These generators were conceived to extract energy from marine currents and to fulfill the following four main features: (i) being valid for operation at depths greater than $40 \mathrm{~m}$, (ii) minimum installation support structure and civil works, (iii) being floating and easily transportable device, and (iv) being fully automated for emersion and immersion maneuvers.

Figure 1 shows the general view of one of the proposed devices. It is composed of a three-fixed pitch blade propeller and a central pod (gondola), and the shaft of the propeller is coupled to an electrical generator by means of a multiplier gearbox. Three main radial and symmetrically distributed columns start at the pod and end at three torpedoes of an approximately cylindrical shape. These torpedoes contain the inner ballast system used to apply hydrostatic forces to the generator. Another of the principal missions of these torpedoes, which are aligned to the direction of the current, is 
TABLE 1: Main features.

\begin{tabular}{lcc}
\hline & A.6.7 model & U1M model (Figure 1) \\
\hline Power (kW) & 600 & 1,000 \\
Stream highest velocity (m/s) & 2.0 & 1.8 \\
Seabed depth $(\mathrm{m})$ & $60 / 100$ & 80 \\
Propeller diameter $(\mathrm{m})$ & 20 & 32 \\
Minimum blade end depth $(\mathrm{m})$ & 15 & 34 \\
Rotor/gear output $(\mathrm{rpm})$ & $12 / 1500$ & $12 / 750$ \\
Structure material & Steel & Steel \\
\hline
\end{tabular}

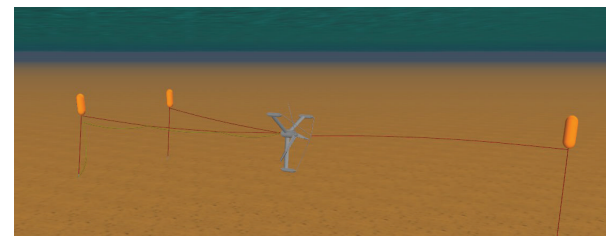

FIGURE 2: Mooring system based on wires and buoys.

to minimize rotations around the main axis of the whole generator by using hydrodynamic force compensation when it is extracting energy and the propeller reaction causes heeling torques. Three structural bars starting at the torpedoes and ending in front of the propeller have been added for mooring purposes.

The main features of two of the conceived generators are briefly presented in Table 1.

Various anchoring or mooring systems can be used to place the generator in the sea at the desired position. Figure 2 shows a simple mooring system based on wires and buoys that allows the device to be positioned at the desired depth. By simply providing the generator with positive floatability, the wires become under strain and the device can be moored in both the desired position and depth and is ready to harness energy.

When employing the emersion maneuver procedure, the device has to evolve from its vertical and submerged operating position and orientation (usually denominated as posture or attitude) to its maintenance and transportation position and orientation (floating horizontally) (see Figure 3). This movement is achieved in three basic sequential steps: (1) by providing the device with zero buoyancy rather than positive buoyancy; (2) by releasing the stern wire; and (3) by managing the water in the ballast tanks.

\section{Dynamic Model}

Obtaining a dynamic model of the device shown previously under three DoFs in order to perform submerged motions is an important step for the simulation and design control algorithms needed to perform an emersion maneuver in a fully automatic closed loop mode. The proposed model is based on the following assumptions (see Figure 4 for details): (i) Only four lumped masses are considered.

(ii) Added masses are constant, well known, and those which are lumped are also considered.

(iii) Vertical translation (one DoF) dynamics and rotation (two DoFs) dynamics are fully uncoupled.

(iv) Viscous friction is modeled as being constant and fully uncoupled.

(v) The device free-surface interaction (considered semisubmergible) is not considered in this paper.

(vi) The influences of residual flow of water, waves, or wind effects are considered as external perturbations in the model.

A fixed reference frame $\mathbf{S}_{0}$ is defined as an orthogonal reference frame whose $z$-axis is vertical. Zero depth $(z=$ 0 ) is computed at the level of the sea (free surface) and the vertical plane $(x, z)$ always contains the center of the generator. Only the depth of the center of the generator is related to this reference frame, and components $x$ and $y$ of the generator with regard to $S_{0}$ are not considered. The other two degrees of freedom (only rotations around axes $x_{G}$ and $y_{G}$ are possible) are defined with regard to the other intermediate reference frame, $S_{G}$, whose origin is at the center gravity of the generator and whose plane $\left(x_{G}, y_{G}\right)$ is placed on an absolutely horizontal plane (parallel to the sea surface). If the generator is positioned with null orientation $\left(\varphi_{x}=0, \theta_{y}=0\right)$, the $x_{G^{-}}$ axis remains perpendicular to the plane of the generator and the $z_{G}$-axis is placed vertically and forms a symmetry axis that is aligned with the mass denoted as $m_{3}$.

If we consider now the actuators, which are responsible for creating the buoyancy forces, they are ideally located at the same positions at which each of these lumped masses is considered to be placed. These actuators will produce vertical component forces only that are obtained as differences between buoyancy forces (really produced by actuators) and forces due to gravity. Each of these masses (denoted and numbered with subindex $i=1,2,3$ ) is formed of both real mass $m_{\mathrm{Gi}}$ and added mass $m_{\mathrm{ADD} i}$ and is

$$
\begin{aligned}
m_{i} & =m_{\mathrm{Gi}}+m_{\mathrm{ADD} i}, \\
f_{i} & =\left(\rho_{W} V_{i}-m_{i}\right) g,
\end{aligned}
$$

where $V_{i}$ is the volume fraction of the generator corresponding to the $i$ th-mass. Under static equilibrium conditions $\left(\rho_{W} V_{i}=m_{i}\right)$, no forces are produced and the generator will remain in its previous state without any kind of motion. In the opposite sense, if motion of the generator is desired, each actuator will produce an incremental force that has to be computed as an increase in either mass or volume (with the opposite sign). For the sake of clarity, volume increments are considered to be responsible for creating buoyancy forces. Consider

$$
f_{i}=+g \rho_{W} \Delta V_{i}
$$



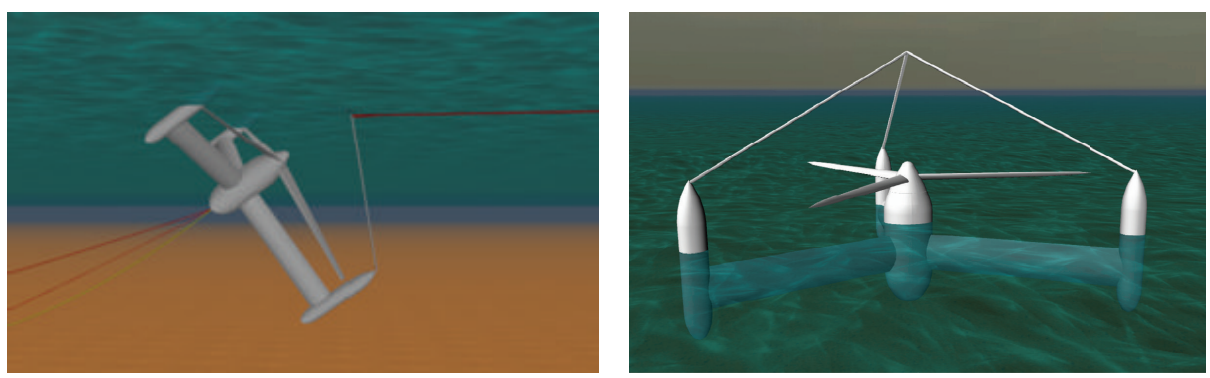

FIGURE 3: Final stages of the emersion maneuver.

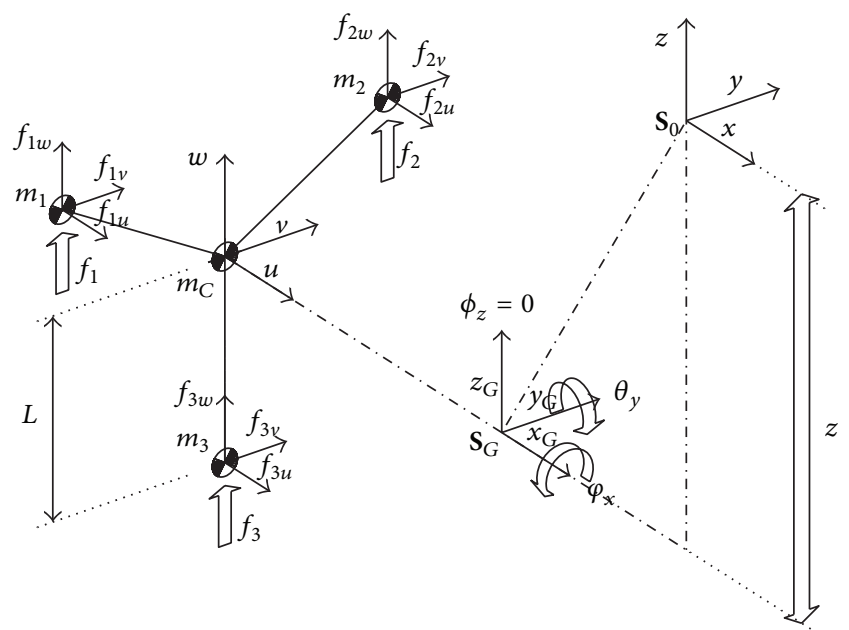

FIGURE 4: Main parameters and magnitudes.

The force vector applied to the generator with regard to the fixed reference frame $S_{0}$ (also with regard to $S_{G}$ ) can be expressed in a matrix form as the sum of all the vertical forces produced by actuators:

$$
\mathbf{F}=\left(\begin{array}{c}
F_{x} \\
F_{y} \\
F_{z}
\end{array}\right)=\left(\begin{array}{c}
0 \\
0 \\
f_{1}+f_{2}+f_{3}
\end{array}\right)
$$

3.1. Forces and Torques Involved. Let us consider a local frame $\mathbf{S}$ placed at the center of the central cylinder of the generator at which its center of gravity is located. This $\mathbf{S}$ frame is defined as follows: the $u$-axis is perpendicular to the plane of the generator, the $w$-axis is located in the opposite direction to the lower mass $m_{3}$ (see Figure 4 ), and the $v$-axis is located at the plane of the generator and forms a right-hand frame. The central mass $m_{\mathrm{CG}}$ is then located at the following local position:

$$
\mathbf{P}_{\mathrm{CLOC}}=\left(\begin{array}{lll}
0 & 0 & 0
\end{array}\right)^{T}
$$

while the three resting lumped masses are located at the center of gravity of each of the three torpedoes with local coordinates (for the sake of simplicity, notations $c \alpha \equiv \cos (\alpha)$ and $s \alpha \equiv \sin (\alpha)$ are used):

$$
\begin{aligned}
& \mathbf{P}_{1 \mathrm{LOC}}=\left(\begin{array}{c}
0 \\
-L s 60^{\circ} \\
L c 60^{\circ}
\end{array}\right), \\
& \mathbf{P}_{2 \mathrm{LOC}}=\left(\begin{array}{c}
0 \\
L s 60^{\circ} \\
L c 60^{\circ}
\end{array}\right), \\
& \mathbf{P}_{3 \mathrm{LOC}}=\left(\begin{array}{c}
0 \\
0 \\
-L
\end{array}\right) .
\end{aligned}
$$

According to the definition of the second frame, $\mathbf{S}_{G}$, the relation of the orientation of both the $\mathbf{S}$ and $\mathbf{S}_{G}$ frames can easily be obtained by computing the rotation matrix that relates the local generator orientation with regard to the $\mathbf{S}_{G}$ reference system. This rotation is the result of composing the next two elementary rotations with regard to the next axes (first horizontal $x_{G}$-axis and then horizontal $y_{G}$-axis basic rotations):

$$
\begin{aligned}
\mathbf{R} & =\mathbf{R}\left(Y, \theta_{y}\right) \cdot \mathbf{R}\left(X, \varphi_{x}\right) \cdot \mathbf{I}^{3 \times 3}, \\
\mathbf{R} & =\left(\begin{array}{ccc}
c \theta_{y} & 0 & s \theta_{y} \\
0 & 1 & 0 \\
-s \theta_{y} & 0 & c \theta_{y}
\end{array}\right) \cdot\left(\begin{array}{ccc}
1 & 0 & 0 \\
0 & c \varphi_{x} & -s \varphi_{x} \\
0 & s \varphi_{x} & c \varphi_{x}
\end{array}\right) \\
& =\left(\begin{array}{ccc}
c \theta_{y} & s \theta_{y} s \varphi_{x} & s \theta_{y} c \varphi_{x} \\
0 & c \varphi_{x} & -s \varphi_{x} \\
-s \theta_{y} & c \theta_{y} s \varphi_{x} & c \theta_{y} c \varphi_{x}
\end{array}\right) .
\end{aligned}
$$


The positions of the three lumped masses with regard to $\mathbf{S}_{G}$ can therefore be written as

$$
\begin{aligned}
& \mathbf{P}_{\mathbf{1}}=\mathbf{R} \cdot \mathbf{P}_{1 \mathrm{LOC}}=\left(\begin{array}{c}
L s \theta\left(-s 60^{\circ} s \varphi_{x}+c 60^{\circ} c \varphi_{x}\right) \\
-L\left(s 60^{\circ} c \varphi_{x}-c 60^{\circ} s \varphi_{x}\right) \\
L c \theta\left(-s 60^{\circ} s \varphi_{x}+c 60^{\circ} c \varphi_{x}\right)
\end{array}\right), \\
& \mathbf{P}_{\mathbf{2}}=\mathbf{R} \cdot \mathbf{P}_{2 \mathrm{LOC}}=\left(\begin{array}{c}
L s \theta\left(s 60^{\circ} s \varphi_{x}+c 60^{\circ} c \varphi_{x}\right) \\
L\left(s 60^{\circ} c \varphi_{x}-c 60^{\circ} s \varphi_{x}\right) \\
L c \theta\left(s 60^{\circ} s \varphi_{x}+c 60^{\circ} c \varphi_{x}\right)
\end{array}\right), \\
& \mathbf{P}_{\mathbf{3}}=\mathbf{R} \cdot \mathbf{P}_{3 \mathrm{LOC}}=\left(\begin{array}{c}
-L s \theta c \varphi_{x} \\
L s \varphi_{x} \\
-L c \theta c \varphi_{x}
\end{array}\right) .
\end{aligned}
$$

Moreover, the application of each of the forces caused by actuators (see forces $f_{1}, f_{2}$, and $f_{3}$ in Figure 4) to their respective centers of gravity on each torpedo leads to a torque vector (with regard to $\mathbf{S}_{G}$ ), which is defined and obtained as follows:

$$
\boldsymbol{\Gamma}=\left(\begin{array}{c}
\Gamma_{x} \\
\Gamma_{y} \\
\Gamma_{z}
\end{array}\right)=\mathbf{P}_{1}\left(\begin{array}{c}
0 \\
0 \\
f_{1}
\end{array}\right)+\mathbf{P}_{2}\left(\begin{array}{l}
0 \\
0 \\
f_{2}
\end{array}\right)+\mathbf{P}_{3}\left(\begin{array}{c}
0 \\
0 \\
f_{3}
\end{array}\right)
$$

And, by substituting (9) in (10), one obtains

$\Gamma$

$$
=\left(\begin{array}{c}
L\left[s 60^{\circ} c \varphi_{x}\left(-f_{1}+f_{2}\right)-c 60^{\circ} s \varphi_{x}\left(f_{1}+f_{2}\right)+s \varphi_{x} f_{3}\right] \\
L s \theta_{y}\left[s 60^{\circ} c \varphi_{x}\left(f_{1}-f_{2}\right)-c 60^{\circ} c \varphi_{x}\left(f_{1}+f_{2}\right)+c \varphi_{x} f_{3}\right] \\
0
\end{array}\right) .
$$

As expected, from (11), it is proven that it is impossible to cause changes in the horizontal direction (rotations about $z_{G^{-}}$ axis or $z$-axis) with only vertical forces.

3.2. Force Conversion. In this subsection, a matrix relation between the forces applied to the generator and a new generalized force vector is proposed. This relation makes it possible to decouple the motions of the generator in the sense that different sets of forces can be applied which then produce independent motions for each of the three degrees of freedom of the generator.

By rearranging (4) and (11), the following relation is easily obtained:

$$
\begin{gathered}
\left(\begin{array}{c}
L\left[s 60^{\circ} c \varphi_{x}\left(-f_{1}+f_{2}\right)-c 60^{\circ} s \varphi_{x}\left(f_{1}+f_{2}\right)+s \varphi_{x} f_{3}\right] \\
L s \theta_{y}\left[s 60^{\circ} c \varphi_{x}\left(f_{1}-f_{2}\right)-c 60^{\circ} c \varphi_{x}\left(f_{1}+f_{2}\right)+c \varphi_{x} f_{3}\right] \\
f_{1}+f_{2}+f_{3}
\end{array}\right) \\
=\left(\begin{array}{c}
\Gamma_{x} \\
\Gamma_{y} \\
F_{z}
\end{array}\right) .
\end{gathered}
$$

By substituting $c 60^{\circ}$ and $s 60^{\circ}$ for their respective values and rearranging (12), the following is attained:

$$
\begin{aligned}
& \left(\begin{array}{ccc}
-\frac{L}{2}\left(\sqrt{3} c \varphi_{x}+s \varphi_{x}\right) & \frac{L}{2}\left(\sqrt{3} c \varphi_{x}-s \varphi_{x}\right) & L s \varphi_{x} \\
\frac{L}{2} s \theta_{y}\left(\sqrt{3} s \varphi_{x}-c \varphi_{x}\right) & -\frac{L}{2} s \theta_{y}\left(\sqrt{3} s \varphi_{x}+c \varphi_{x}\right) & L s \theta_{y} c \varphi_{x} \\
1 & 1 & 1
\end{array}\right) \\
& \cdot\left(\begin{array}{l}
f_{1} \\
f_{2} \\
f_{3}
\end{array}\right)=\left(\begin{array}{c}
\Gamma_{x} \\
\Gamma_{y} \\
F_{z}
\end{array}\right) .
\end{aligned}
$$

Or in a compact form,

$$
\Lambda\left(\varphi_{x}, \theta_{y}\right) \cdot \mathbf{F}_{G}=\boldsymbol{\tau},
$$

where $\mathbf{F}_{G}=\left(\begin{array}{lll}f_{1} & f_{2} & f_{3}\end{array}\right)^{T}$ denotes the buoyancy forces provided by all the actuators, $\boldsymbol{\tau}=\left(\begin{array}{lll}\Gamma_{x} & \Gamma_{y} & F_{z}\end{array}\right)^{T}$ denotes the proposed vector of generalized forces, and $\boldsymbol{\Lambda}$ denotes the matrix that relates $\boldsymbol{\tau}$ to $\mathbf{F}_{G}$, which depends on $\varphi_{x}$ and $\theta_{y}$ according to (13) and (14).

In (14), vector $\boldsymbol{\tau}$ is conceived as the uncoupled generalized forces vector applied to the center of the generator, so the first component will produce a torque that is aligned to the $x_{G^{-}}$ axis and the second component will produce a torque that is aligned to the $y_{G}$-axis while the third component will produce a force that is aligned to the $z$-axis.

3.3. Dynamic Submodel for Only Vertical Movement. The dynamics of an underwater body that is considered to be a rigid body when it performs vertical motions is well known (see [35-37], a.e.). As was seen in the previous section, depth $z$ is the only variable of position that can be controlled. Using the same notation given in the previous subsection and under hypothesis shown above, the next dynamic model is proposed for only vertical motions (the effects resulting from freesurface interaction are not computed):

$$
F_{z}=\left(m_{G}+m_{\mathrm{ADD}}\right) \cdot \ddot{z}+\underbrace{|\dot{z}| \cdot v_{z} \cdot \dot{z}}_{F_{z v}(\dot{z})}
$$

where $m_{G}=m_{\mathrm{CG}}+m_{1 G}+m_{2 G}+m_{3 G}$ denotes the total mass of the generator and is decomposed into the mass of the central cylinder $m_{\mathrm{CG}}$ and the three masses of each torpedo. For the sake of simplicity, the term $m_{\mathrm{ADD}}$ denotes the total added mass resulting from the motion in a viscous fluid (see [38]) which is considered to be constant and well known. A quadratic and speed opposite friction term is computed as $F_{z v}(\dot{z})=|\dot{z}| \cdot v_{z} \cdot \dot{z}$, where $v_{z}$ is also considered to be constant.

3.4. Dynamic Submodel for Only Rotation Movements. In this subsection, the Lagrange formulation allows us to obtain the equations of motion for the generator provided with only rotation movements. The two rotation angles $\varphi_{x}$ and $\theta_{y}$ around their respective $x_{G}$-axis and $y_{G}$-axis are taken as generalized rotation coordinates $\mathbf{q}_{\mathbf{R}}=\left(\varphi_{x} \theta_{y}\right)^{T}$ for rotation purposes solely. For the compotation of the Lagrange function, the kinetic and potential energies are computed beforehand. 
3.4.1. Local Inertia Matrix. The inertia matrix in local coordinates is obtained from the lumped mass distribution employed. Because the general rotation motion is of dimension 3 , the inertia matrix results are of dimensions $3 \times 3$ :

$$
\mathbf{J}_{\mathrm{LOC}}=\left(\begin{array}{ccc}
I_{x x} & P_{x y} & P_{x z} \\
P_{y x} & I_{y y} & P_{y z} \\
P_{z x} & P_{z y} & I_{z z}
\end{array}\right)
$$

which satisfies that it is a symmetrical and positive definite matrix. This implies that eig $\left(\mathrm{J}_{\mathrm{LOC}}\right)>0, P_{x y}=P_{y x}, P_{x z}=P_{z x}$, and $P_{y z}=P_{z y}$. In our case, the inertia matrix incorporates the effects of the added masses and takes the form (details of how the coefficients in this inertia matrix were obtained can be found in Appendix A):

$$
\begin{aligned}
& \mathrm{J}_{\mathrm{LOC}} \\
& =\left(\begin{array}{ccc}
m_{1}+m_{2}+m_{3} & 0 & 0 \\
0 & \frac{m_{1}+m_{2}}{4}+m_{3} & \frac{\sqrt{3}\left(m_{2}-m_{1}\right)}{4} \\
0 & \frac{\sqrt{3}\left(m_{2}-m_{1}\right)}{4} & \frac{3\left(m_{1}+m_{2}\right)}{4}
\end{array}\right) \\
& \cdot L^{2} .
\end{aligned}
$$

As in the case shown above, each of the masses considered is computed as the sum of its real mass fraction and its added mass, as seen in (1). It will be observed that if $m_{1}=$ $m_{2}$, the generator becomes fully statically and dynamically equilibrated with all of its null value inertia products $\left(P_{x y}=\right.$ $\left.P_{x z}=P_{y z}=0\right)$.

3.4.2. Computation of the Rotation Kinetic Energy. The kinetic energy of the generator when only rotations over first the $x_{G}$-axis and then the $y_{G}$-axis are considered is given by

$$
\begin{aligned}
& \mathbf{K}=\frac{1}{2}\left(\Omega_{x} \quad \Omega_{y} \quad \Omega_{z}=0\right) \cdot \mathbf{R}^{T}\left(\varphi_{x}, \theta_{y}\right) \\
& \cdot\left(\begin{array}{ccc}
I_{x x} & 0 & 0 \\
0 & I_{y y} & P_{y z} \\
0 & P_{y z} & I_{z z}
\end{array}\right) \cdot \mathbf{R}\left(\varphi_{x}, \theta_{y}\right) \cdot\left(\begin{array}{c}
\Omega_{x} \\
\Omega_{y} \\
\Omega_{z}=0
\end{array}\right)
\end{aligned}
$$

with $\Omega_{x}, \Omega_{y}$, and $\Omega_{z}$ being the absolute rotational velocities of the generator with respect to $S_{G}$.

By substituting (8) and (17), one obtains

$$
\begin{aligned}
\mathbf{K} & =\frac{1}{2}\left[\Omega_{x}^{2}\left(I_{x x} c^{2} \theta_{y}+I_{z z} s^{2} \theta_{y}\right)\right. \\
& +2 \Omega_{x} \Omega_{y}\left[\left(I_{x x}-I_{z z}\right) s \theta_{y} c \theta_{y} s \varphi_{x}-P_{y z} s \theta_{y} c \varphi_{x}\right] \\
& +\Omega_{y}^{2}\left[\left(I_{x x} s^{2} \theta_{y}+I_{z z} c^{2} \theta_{y}\right) s^{2} \varphi_{x}+I_{y y} c^{2} \varphi_{x}\right. \\
& \left.\left.+2 P_{y z} c \theta_{y} s \varphi_{x} c \varphi_{x}\right]\right] .
\end{aligned}
$$

3.4.3. Computation of the Rotation Potential Energy. In order to control the generator, it is necessary to obtain operation conditions that force the generator to be at an equilibrium point. Neutral buoyancy is therefore required $\left(m_{G}=\rho_{w} V\right)$, and the total potential energy thus has a zero value under this condition

$$
\mathbf{U}=0 \text {. }
$$

3.4.4. Lagrange Formulation. The kinetic and the potential energy computed previously are used, and the Lagrange function of the generator with only rotation motions with regard to the $x_{G}$-axis and $y_{G}$-axis is

$$
\mathbf{L}=\mathbf{K}-\mathbf{U}=\mathbf{K} \text {. }
$$

Lagrange's equations are then expressed by

$$
\frac{d}{d t}\left(\frac{\partial \mathbf{L}}{\partial \dot{\mathbf{q}}_{\mathbf{R}}}\right)-\frac{\partial \mathbf{L}}{\partial \mathbf{q}_{\mathbf{R}}}=\tau,
$$

where $\tau$ is the sum of external torques, which are the control torques $\left(\begin{array}{lll}\Gamma_{x} & \Gamma_{y}\end{array}\right)^{T}$ and the friction terms that have been modeled as a function $\mathbf{F}_{\mathbf{v}}\left(\dot{\mathbf{q}}_{\mathbf{R}}\right)$ with $\boldsymbol{v}_{\mathbf{R}}=\left(\begin{array}{ll}v_{\varphi x} & v_{\theta y}\end{array}\right)^{T}$. The following expression is obtained (see the coefficients of $\mathbf{M}_{\mathbf{R}}$ and $\mathbf{C}_{\mathbf{R}}$ in Appendix B):

$$
\mathbf{M}_{\mathbf{R}}\left(\mathbf{q}_{\mathbf{R}}\right) \cdot \ddot{\mathbf{q}}_{\mathbf{R}}+\mathbf{C}_{\mathbf{R}}\left(\mathbf{q}_{\mathbf{R}}, \dot{\mathbf{q}}_{\mathbf{R}}\right)=\left(\begin{array}{c}
\Gamma_{x} \\
\Gamma_{y}
\end{array}\right)-\underbrace{\left|\dot{\mathbf{q}}_{\mathbf{R}}^{T}\right| \cdot \boldsymbol{v}_{\mathbf{R}} \cdot \dot{\mathbf{q}}_{\mathbf{R}}}_{\mathbf{F}_{\mathbf{v}}\left(\dot{\mathbf{q}}_{\mathbf{R}}\right)}
$$

in which $\mathbf{q}_{\mathbf{R}}$ denotes the generalized rotation coordinates, $\mathbf{q}_{\mathbf{R}}=\left(\varphi_{x} \theta_{y}\right)^{T}$ with regard to $\mathbf{S}_{\mathbf{G}}$ frame, $\mathbf{M}_{\mathbf{R}}$ are the inertia terms which are $\mathbf{q}_{\mathbf{R}}$ dependent, positive definite, and symmetrical, and $\mathbf{C}_{\mathbf{R}}$ includes both the centrifugal and Coriolis effects. A term $\boldsymbol{v}_{\mathbf{R}}=\left(v_{\varphi x} v_{\theta y}\right)^{T}$ allows the friction terms to be modeled in a similar way to that occurring in (15).

3.5. Proposed Dynamic Model. The complete proposed dynamic model can be obtained by joining (13), (15), and (23) and expressed in a compact form as

$$
\mathbf{M}(\mathbf{q}) \cdot \ddot{\mathbf{q}}+\mathbf{C}(\mathbf{q}, \dot{\mathbf{q}})=\boldsymbol{\Lambda}(\mathbf{q}) \cdot \mathbf{F}_{G}-\left|\dot{\mathbf{q}}^{T}\right| \cdot \boldsymbol{v} \cdot \dot{\mathbf{q}},
$$

where the complete vector of generalized coordinates $\mathbf{q}$ is

$$
\mathbf{q}=\left(\begin{array}{c}
\mathbf{q}_{\mathbf{R}} \\
z
\end{array}\right)
$$

and matrices $\mathbf{M}, \mathbf{C}$, and $\boldsymbol{v}$ are

$$
\begin{aligned}
\mathbf{M}(\mathbf{q}) & =\left(\begin{array}{cc}
\mathbf{M}_{\mathbf{R}}\left(\mathbf{q}_{\mathbf{R}}\right) & \mathbf{0}^{\mathbf{2 \times 1}} \\
\mathbf{0}^{\mathbf{1} \times \mathbf{2}} & m_{G}+m_{\mathrm{ADD}}
\end{array}\right), \\
\mathbf{C}(\mathbf{q}, \dot{\mathbf{q}}) & =\left(\begin{array}{c}
\mathbf{C}_{\mathbf{R}}\left(\mathbf{q}_{\mathbf{R}}, \dot{\mathbf{q}}_{\mathbf{R}}\right) \\
0
\end{array}\right), \\
\boldsymbol{v} & =\left(\begin{array}{c}
v_{\mathbf{R}}=\left(\begin{array}{c}
v_{\varphi x} \\
v_{\theta y}
\end{array}\right) \\
v_{z}
\end{array}\right) .
\end{aligned}
$$




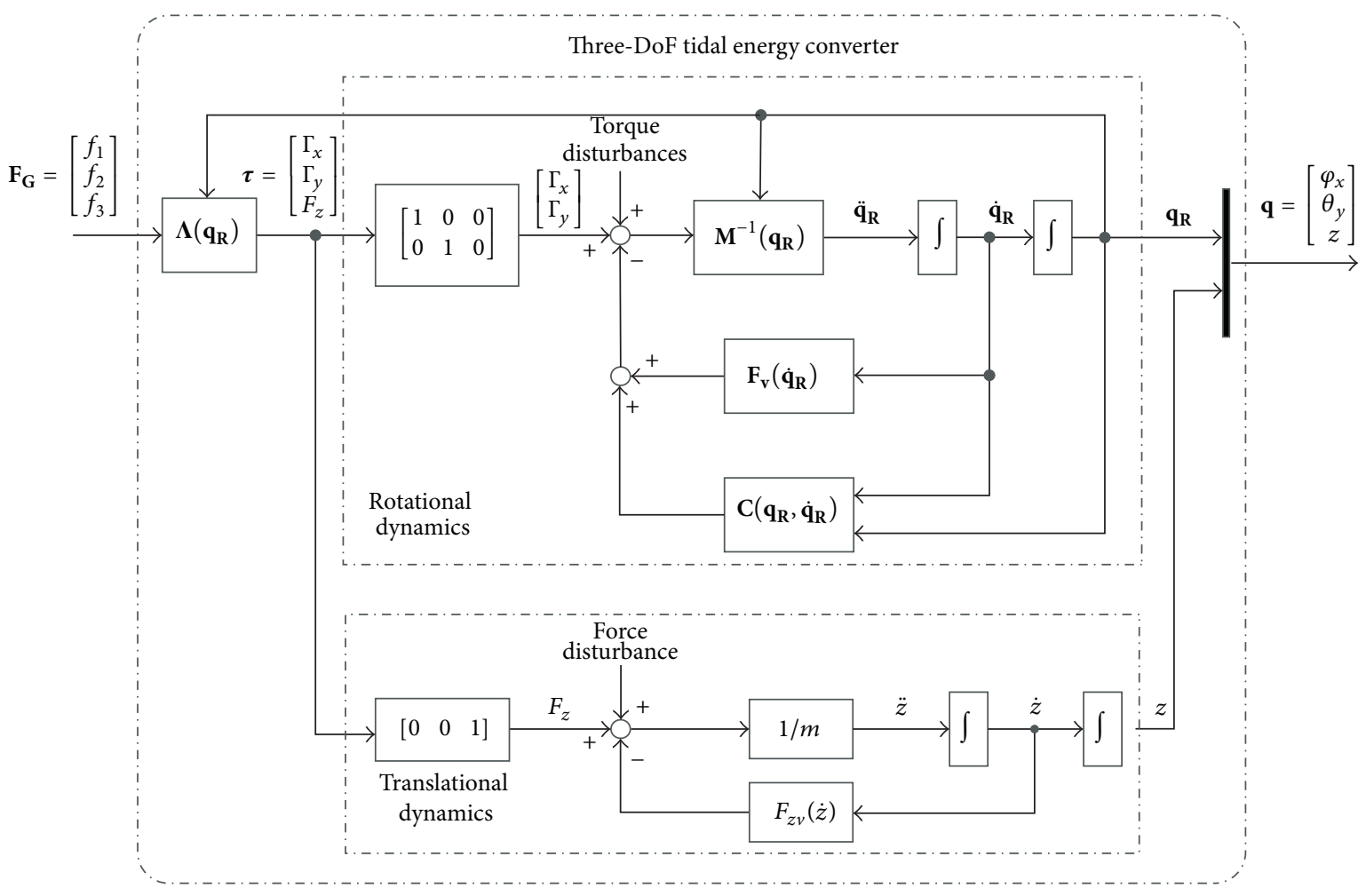

Figure 5: Proposed dynamic model.

Figure 5 depicts the proposed dynamic model, along with the two submodels presented above. The set of input signals are the buoyancy forces produced by actuators $\mathbf{F}_{G}=$ $\left(\begin{array}{lll}f_{1} & f_{2} & f_{3}\end{array}\right)^{T}$ while the output measurable signals are the twodegree-of-freedom orientation angles together with the generator depth denoted previously as a vector of the generalized coordinates $\mathbf{q}=\left(\begin{array}{lll}\varphi_{x} & \theta_{y} & z\end{array}\right)^{T}$.

\section{Uncoupled Multivariable PD Control}

The three-degree-of-freedom model proposed clearly exhibits a nonlinear, time variant, and multivariable uncoupled behavior. A new and simple control scheme based on the following three stages is proposed: (i) an uncoupled term that allows the generalized control torques to be converted into forces applied to each of the torpedoes (by means of actuators), (ii) a nonlinear uncoupling model-based term that ideally cancels out the nonlinearities of the system caused by centrifugal and Coriolis torques, and (iii) a multivariable diagonal proportional-derivative (PD) controller.

4.1. Uncoupling Matrix. The determinant of the matrix $\Lambda(\mathbf{q})$ defined in (13) and (14) is

$$
|\boldsymbol{\Lambda}(\mathbf{q})|=\frac{3 \sqrt{3}}{2} L^{2} s \theta_{y} .
$$

This determinant makes matrix $\boldsymbol{\Lambda}(\mathbf{q})$ nonsingular, with the exception of $\theta_{y}=0$ which corresponds to the normal operation orientation. In other words, if the generator is placed in a fully vertically position, it is not possible to turn around the $y_{G}$-axis using vertical forces alone. In order to avoid this singularity, the position of the third actuator can be displaced a small distance $\delta_{x}$ along the local $u$-axis, thus resulting in its new local position at (see Figure 6)

$$
\mathbf{P}_{3 \mathrm{LOC}}=\left(\begin{array}{c}
\delta_{x}>0 \\
0 \\
-L
\end{array}\right) \text {. }
$$

For relatively small values of $\delta_{x}$ the angle of the new plane that conforms to the three points of application of forces with regard to the plane of the generator is (see Figure 6)

$$
\Delta \theta_{y}=\tan ^{-1}\left(\frac{2 \delta_{x}}{3 L}\right) \approx \frac{2 \delta_{x}}{3 L}
$$

and the range of the angle $\theta_{y}$ is $-\left(\pi / 2+2 \delta_{x} / 3 L\right) \leq \theta_{y} \leq$ $-2 \delta_{x} / 3 L$ rather than $-\pi / 2 \leq \theta_{y} \leq 0$, thus avoiding the singularity of matrix $\boldsymbol{\Lambda}(\mathbf{q})$ in (29). For the angle $\varphi_{x}$ is considered range $-\pi / 2 \leq \varphi_{x} \leq \pi / 2$. 


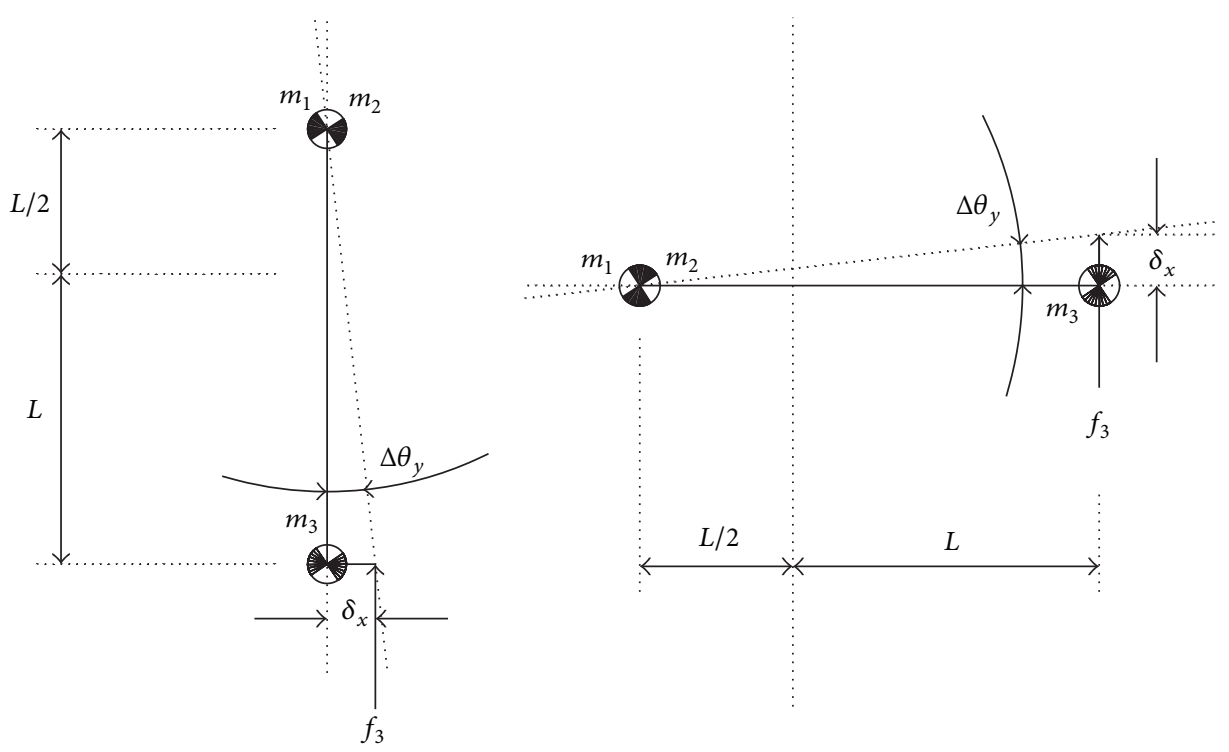

FIGURE 6: Singularity avoidance.

Then, after avoiding the singular values of the angle $\theta_{y}$, the inverse of matrix $\Lambda(\mathbf{q})$ can be obtained symbolically, yielding

$$
\begin{aligned}
& \Lambda^{-1}(\mathbf{q}) \\
& =\left(\begin{array}{ccc}
-\frac{\left(\sqrt{3} c \varphi_{x}+s \varphi_{x}\right)}{3 L} & -\frac{\left(c \varphi_{x}-\sqrt{3} s \varphi_{x}\right)}{3 L s \theta_{y}} & \frac{1}{3} \\
\frac{\left(\sqrt{3} c \varphi_{x}-s \varphi_{x}\right)}{3 L} & -\frac{\left(c \varphi_{x}+\sqrt{3} s \varphi_{x}\right)}{3 L s \theta_{y}} & \frac{1}{3} \\
\frac{2 c \varphi_{x}}{3 L} & \frac{1}{3 L s \theta_{y}} & \frac{1}{3}
\end{array}\right) .
\end{aligned}
$$

4.2. Proposed Control System. The existence of the inverse of matrix $\boldsymbol{\Lambda}(\mathbf{q})$ into (29), (32) makes it possible to handle the generator in an uncoupled mode and even in open loop, by simply obtaining the forces to be applied by actuators $\mathbf{F}_{G}$ from a set of desired and uncoupled generalized forces $\boldsymbol{\tau}$ for nonsingular values of $\theta_{y}$. The existence of external disturbances and model uncertainties between the real matrix $\Lambda(\mathbf{q})$ and the computed matrix $\widehat{\Lambda}(\mathbf{q})$ requires the addition of closed loop controllers.

Equations (24) and (32) provide the following control system, which is proposed for a given desired reference $\mathbf{q}_{d}=$ $\left(\begin{array}{lll}\varphi_{x d} & \theta_{y d} & z_{d}\end{array}\right)^{T}$ and which can be time dependent:

$$
\mathbf{F}_{G}=\widehat{\Lambda}(\mathbf{q})^{-1} \cdot\left[\widehat{\mathbf{C}}(\mathbf{q}, \dot{\mathbf{q}})-\mathbf{K}_{\mathbf{D}} \dot{\mathbf{q}}+\mathbf{K}_{\mathbf{P}}\left(\mathbf{q}_{d}-\mathbf{q}\right)\right],
$$

where $\widehat{\Lambda}(\mathbf{q})$ and $\widehat{\mathbf{C}}(\mathbf{q}, \dot{\mathbf{q}})$ denote their respective computed matrices as a function of the measured output signals $\mathbf{q}$. The complete dynamics of the system controller, under the assumption of perfect cancellation, that is, $\widehat{\mathbf{C}}(\mathbf{q}, \dot{\mathbf{q}})=\mathbf{C}(\mathbf{q}, \dot{\mathbf{q}})$ and $\widehat{\Lambda}(\mathbf{q})=\Lambda(\mathbf{q})$, results in

$$
\mathbf{M}(\mathbf{q}) \cdot \ddot{\mathbf{q}}+\mathbf{K}_{\mathbf{D}} \cdot \dot{\mathbf{q}}+\mathbf{K}_{\mathbf{P}} \cdot \mathbf{q}=\mathbf{K}_{\mathbf{P}} \cdot \mathbf{q}_{d} .
$$

Since the inertia matrix $\mathbf{M}(\mathbf{q})$ is defined according to (26) and the coefficients shown in Appendix A, it is a bounded symmetric and positive definite matrix which satisfies that $\lambda_{\text {min }} \mathbf{I}^{\mathbf{3} \times \mathbf{3}} \leq \mathbf{M}(\mathbf{q}) \leq \lambda_{\mathrm{MAX}} \mathbf{I}^{\mathbf{3 \times 3}}$ for all $\mathbf{q}$. Upon considering a mean inertia matrix as

$$
\overline{\mathbf{M}}=\frac{\left.\mathbf{M}(\mathbf{q})\right|_{\text {MAX }}+\left.\mathbf{M}(\mathbf{q})\right|_{\min }}{2}
$$

the closed loop controlled system with mean matrix $\overline{\mathbf{M}}$ exhibits fully decoupled multivariable linear dynamics defined by

$$
\ddot{\mathbf{q}}+\overline{\mathbf{M}}^{-1} \cdot \mathbf{K}_{\mathbf{D}} \cdot \dot{\mathbf{q}}+\overline{\mathbf{M}}^{-1} \cdot \mathbf{K}_{\mathbf{P}} \cdot \mathbf{q}=\overline{\mathbf{M}}^{-1} \cdot \mathbf{K}_{\mathbf{P}} \cdot \mathbf{q}_{d}
$$

and three independent single input/single output (SISO) PD controllers with derivative in the feedback loops can be tuned by choosing diagonal matrices of gains $\mathbf{K}_{\mathbf{D}}=$

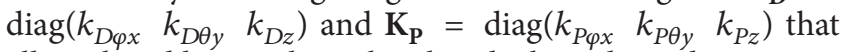
allow closed loop poles to be placed where desired. Equation (36) can then be analyzed as three SISO independent closed loop systems for any of the three degrees of freedom of the generator.

Figure 7 depicts the proposed control scheme. The uncoupling matrix and the linearization term that compensates the centrifugal and Coriolis torques are clearly shown, together with an uncoupled three-dimensional diagonal PD controller with derivative component from only the output signal as proposed in (33).

\section{Numerical Simulations Results}

The proposed dynamic model (24) and the controller (33), presented above, were used to carry out numerical simulations in order to demonstrate the goodness of both the dynamic model and the proposed control in terms of generator controllability and ability to perform emersion and immersion maneuvers with only passive buoyancy 


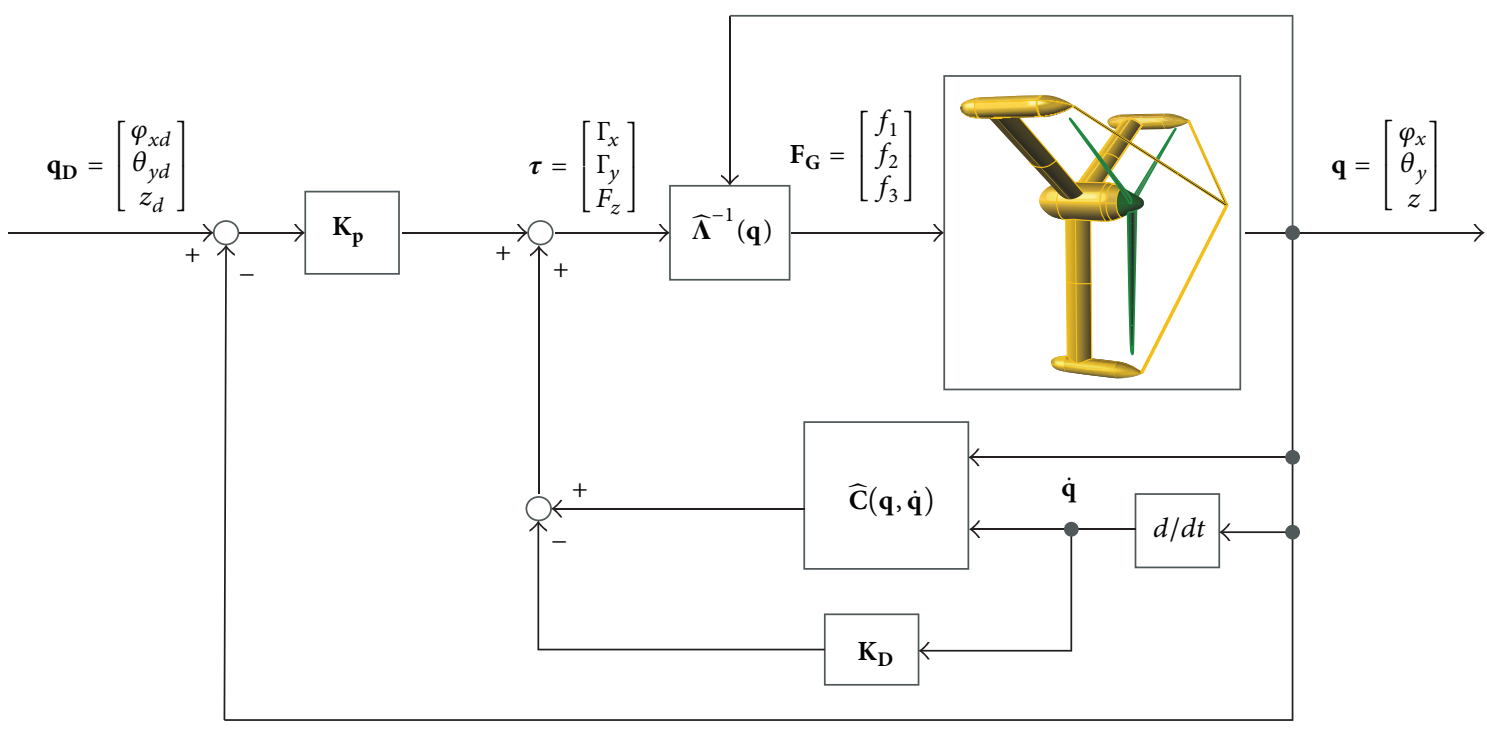

Figure 7: Proposed control system.

TABLE 2: Main parameters for simulation (per each of the cylindrical torpedoes).

\begin{tabular}{lcc}
\hline & Nominal value & Units \\
\hline Number of torpedoes & 3 & \\
Torpedo angle distribution & $0,2 \pi / 3,4 \pi / 3$ & $\mathrm{rad}$ \\
Length & 0.608 & $\mathrm{~m}$ \\
Diameter & 0.200 & $\mathrm{~m}$ \\
Volume & 0.191 & $\mathrm{~m}^{3}$ \\
Real mass & 19.1 & $\mathrm{~kg}$ \\
Added mass & 15.3 & $\mathrm{~kg}$ \\
Friction term & 79.4 & $\mathrm{~N} \cdot \mathrm{s}^{2} \cdot \mathrm{m}^{-2}$ \\
$G$ & 9.81 & $\mathrm{~m} \cdot \mathrm{s}^{-2}$ \\
$\rho_{w}$ & 1,000 & $\mathrm{Kg} \cdot \mathrm{m}^{-3}$ \\
$L$ & 0.400 & $\mathrm{~m}$ \\
\hline
\end{tabular}

forces. Simulations were performed in the Mathworks $@$ Simulink/Matlab environment and developed for a set of parameters that corresponds to a lab prototype that is being built for further experimental validation. Its main values are shown in Table 2.

The main reason for using a laboratory prototype is the high economic cost of a real scale device. The research group has successfully tested a 1:10 scale prototype [39] in a real protected sea environment. From these trials, the high acceleration values observed in open loop in some of the time intervals during the execution of the emersion maneuvers performed (see Figure 22 in [39]) could damage the rotor blades making necessary the study of the proposed closed loop control.

The first set of numerical simulations was performed on step reference signals with nominal parameters of the added mass and the friction term in order to validate both the desired transient and steady state responses and there were no coupled dynamics among the independent movements $\left(\varphi_{x}, \theta_{y}\right.$, and $\left.z\right)$ that the generator can perform. Figures 8 to 10 illustrate the time responses of each of the independent closed loop systems of each of the degrees of freedom of the generator when the following step reference signals were used (units in rad for angles, $\mathrm{m}$ for depth, resp.):

$$
\begin{gathered}
\varphi_{x d} \text { from } 0 \text { to } \frac{\pi}{3} \text { at } t=10 \mathrm{~s}, \\
\theta_{y d} \text { from }-0.05 \text { to }-\frac{\pi}{2} \text { at } t=20 \mathrm{~s}, \\
z_{d} \text { from }-20 \text { to }-1 \text { at } t=20 \mathrm{~s} .
\end{gathered}
$$

The PD controller gains for each of the loops were tuned using the nominal value of matrix $\overline{\mathbf{M}}$ in order to obtain closed loop double poles placed at $p_{1,2}=-0.3 \mathrm{rad} / \mathrm{s}$ and a unit gain that will guarantee critically damped second-order dynamics. For a given fully uncoupled open loop dynamic (second-order and type one system) of the form $G_{i}(s)=A_{i} /\left(s \cdot\left(s+B_{i}\right)\right)$ and a desired closed loop dynamic of the form $M_{i}(s)=p_{1,2}^{2} /\left(s^{2}+\right.$ $\left.2 p_{1,2} s+p_{1,2}^{2}\right)$, for $i=\varphi_{x}, \theta_{y}, z$, the gains of the PD controllers are easily obtained as $k_{P i}=p_{1,2}^{2} / A_{i}$ and $k_{D i}=\left(2 p_{1,2}-B_{i}\right) / A_{i}$. Actuator saturation is not considered here. Gaussian noises from a normal distribution $N\left(0,3 e^{-3}\right)$ were added to each of the output signals, and first-order filters were used to obtain the time derivative of the output signals as $\mathscr{L}(d / d t)=s \approx$ $s /(0.001 s+1)$, where $\mathscr{L}$ denotes the Laplace transform.

Figures 8-10 depict that the system performs extremely well with uncoupled dynamics, unit gain, and the desired setting time response with no overshoot (the effect of the $\theta_{y d}$ transition at $t=20 \mathrm{~s}$ and the real $\theta_{y}$ response at $t \geq 20 \mathrm{~s}$ is not perceived in the time evolution of the response of $\varphi_{x}$ ).

The second set of simulations were carried out assuming nonnominal terms (increments of $\pm 20 \%$ of the nominal added mass term and null Coriolis and centripetal compensation term). Additionally, the actuators were saturated to $\pm 5 \mathrm{~N}$, thus simulating a more realistic scenario. Due to the feedback robustness provided by the PD controller 


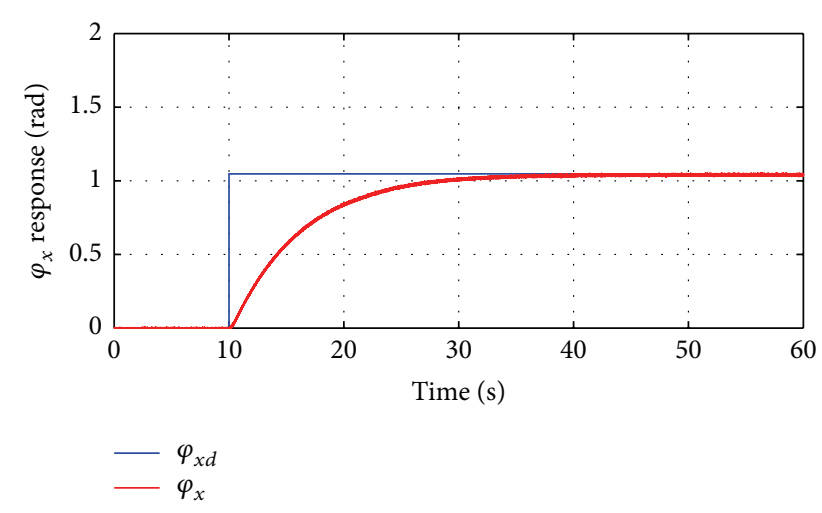

FIGURE 8: Step response of $\varphi_{x}(t)$ from 0 to $\pi / 3$ rad with transition time at $t=10 \mathrm{~s}$.

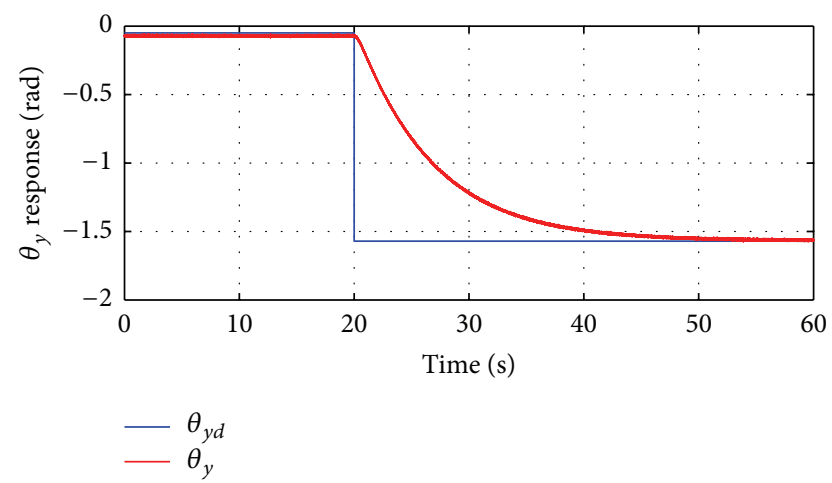

FIGURE 9: Step response of $\theta_{y}(t)$ from -0.05 to $-\pi / 2 \mathrm{rad}$ with transition time at $t=20 \mathrm{~s}$.

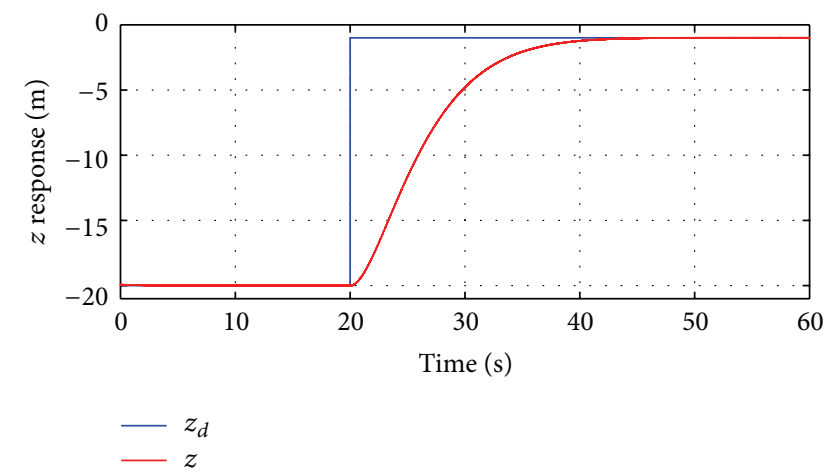

FIGURE 10: Step response of $z(t)$ from -20 to $-1 \mathrm{~m}$ with transition time at $t=20 \mathrm{~s}$.

(see [40], a.e.), the time responses were very similar to the nominal responses shown in Figures 8-10. Figures 11-13 depict the evolution of the errors between the nonnominal and the nominal responses. In these figures is clearly observed the coupling effect at instants $t=10 \mathrm{~s}$ and $t=20 \mathrm{~s}$ when transitions occur due to step signals.

The third set of simulations was carried out using synchronous smooth time trigonometric sixth-order Strajectories (see [41, 42]) with starting and ending times of $t_{0}=20$ to $t_{f}=120 \mathrm{~s}$, respectively, for the orientation

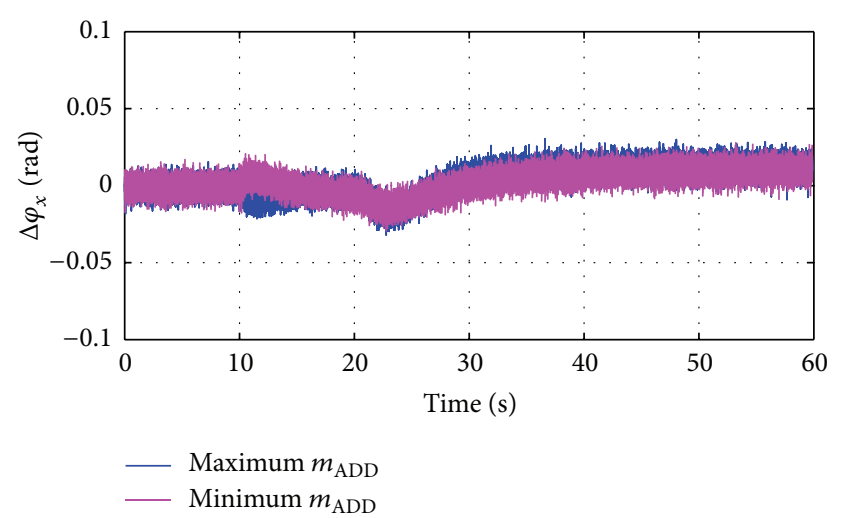

FIGURE 11: Error between $\varphi_{x}$ time responses with nonnominal $m_{\mathrm{ADD}}$ and null Coriolis and centripetal compensation terms.

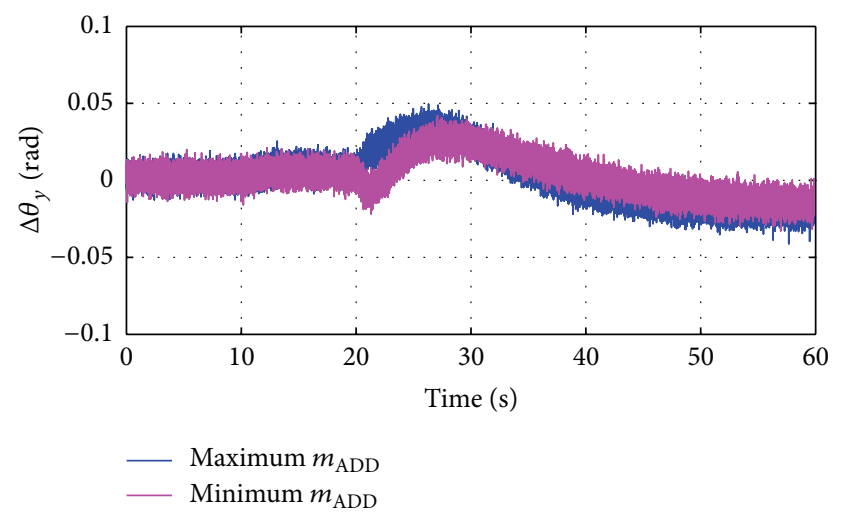

FIGURE 12: Error between $\theta_{y}$ time responses with nonnominal $m_{\mathrm{ADD}}$ and null Coriolis and centripetal compensation terms.

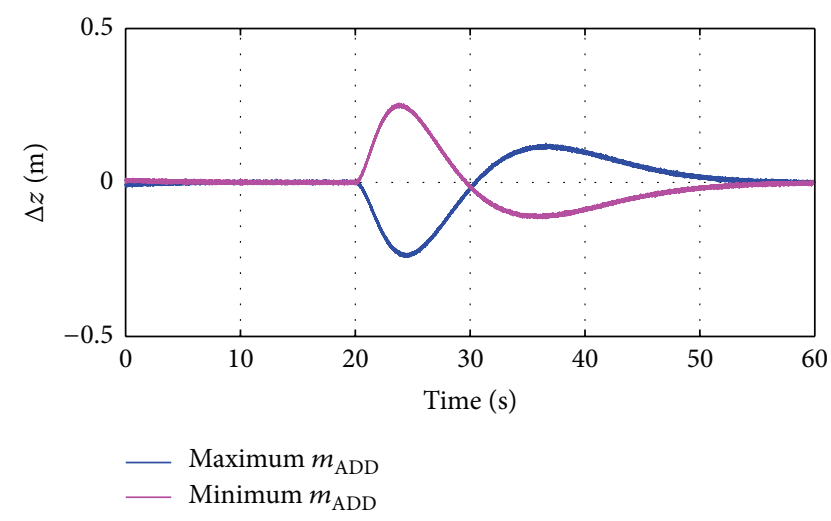

FIGURE 13: Error between $z$ time responses with nonnominal $m_{\mathrm{ADD}}$ and null Coriolis and centripetal compensation terms.

references, and $t_{0}=20$ to $t_{f}=220 \mathrm{~s}$ for the depth reference with the maximum velocities shown below

$$
\begin{aligned}
& \varphi_{x d} \text { from } 0 \text { to } \frac{\pi}{3} \mathrm{rad} \quad\left|\dot{\varphi}_{x d}\right|_{\mathrm{MAX}}=0.0125 \mathrm{rad} / \mathrm{s}, \\
& \theta_{y d} \text { from }-0.05 \text { to }-\frac{\pi}{2} \mathrm{rad} \\
& \qquad\left|\dot{\theta}_{y d}\right|_{\mathrm{MAX}}=0.0175 \mathrm{rad} / \mathrm{s}, \\
& z_{d} \text { from }-20 \text { to }-1 \mathrm{~m} \quad\left|\dot{z}_{d}\right|_{\mathrm{MAX}}=0.125 \mathrm{~m} / \mathrm{s} .
\end{aligned}
$$




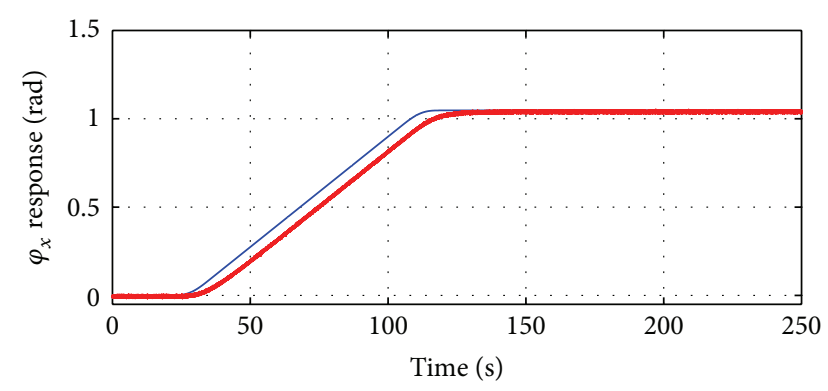

$-\varphi_{x d}$

FIGURE 14: Reference S-trajectory $\varphi_{d x}(t)$ and time response $\varphi_{x}(t)$.

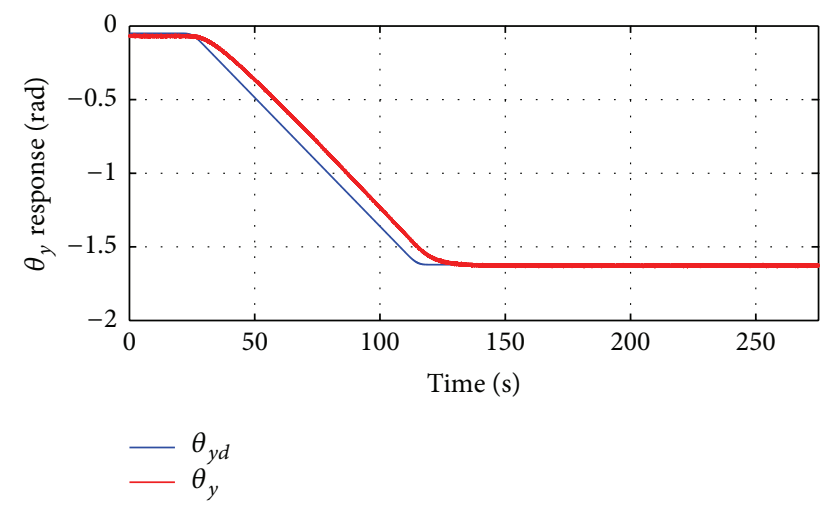

FIGURE 15: Reference S-trajectory $\theta_{d y}(t)$ and time response $\theta_{y}(t)$.

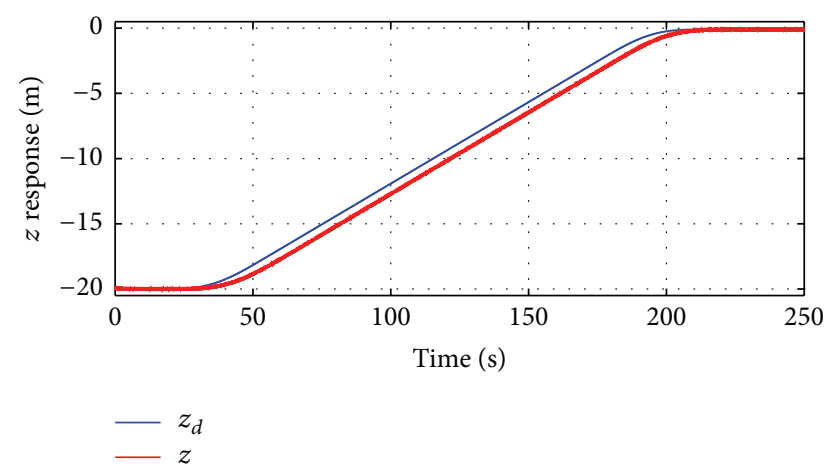

FIgURE 16: Reference S-trajectory $z_{d}(t)$ and time response $z(t)$.

Figures 13-16 illustrate that the generator exhibits a good correspondence with both the desired responses and the simulated responses obtained.

Finally, the fourth set of simulations corresponds to a complete sequence of sixth-order S-trajectories to achieve an emersion maneuver. Figures 17 to 19 depict both the references and the time responses obtained for each of the independent movements of the generator. The desired sequence is defined below (units in rad for angles, $m$ for depth):

$$
\begin{aligned}
\text { from } \mathbf{q}_{d 0}=\left(\begin{array}{c}
0 \\
-0.05 \\
-20
\end{array}\right) \text { to } \mathbf{q}_{d 1}=\left(\begin{array}{c}
0 \\
-0.05 \\
-20
\end{array}\right) \\
t_{0}=0 \text { to } t_{1}=25 \mathrm{~s}
\end{aligned}
$$

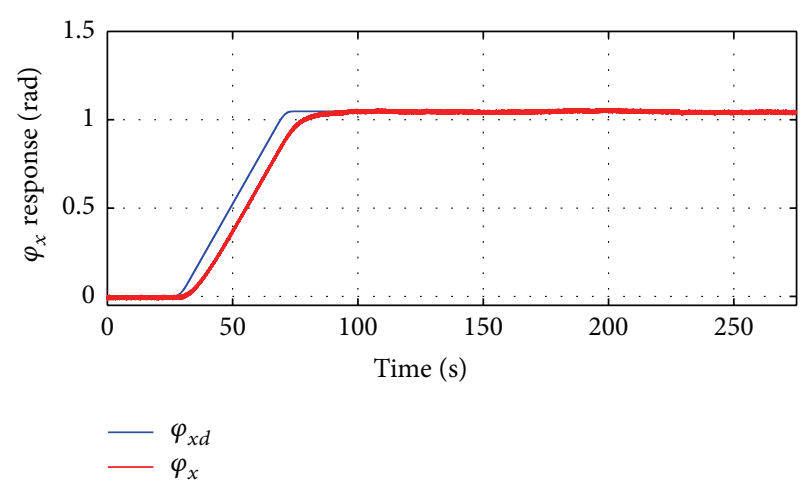

FIGURE 17: Emersion maneuver reference $\varphi_{d x}(t)$ and time response $\varphi_{x}(t)$.

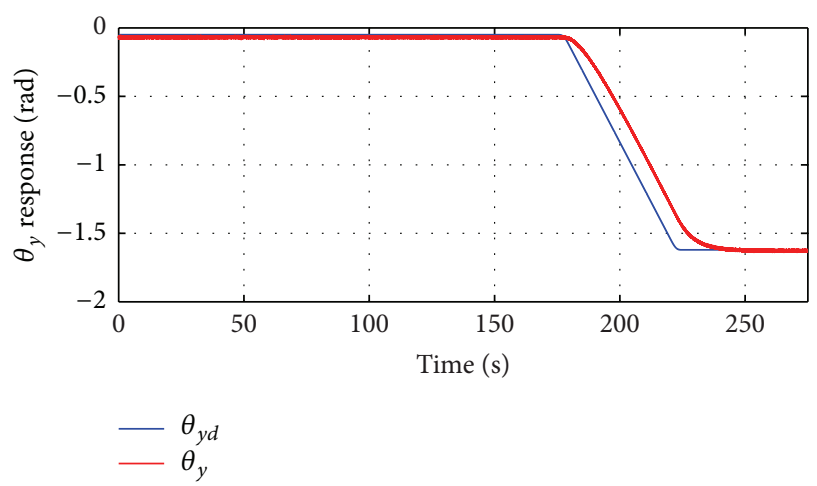

FIGURE 18: Emersion maneuver reference $\theta_{d y}(t)$ and time response $\theta_{y}(t)$.

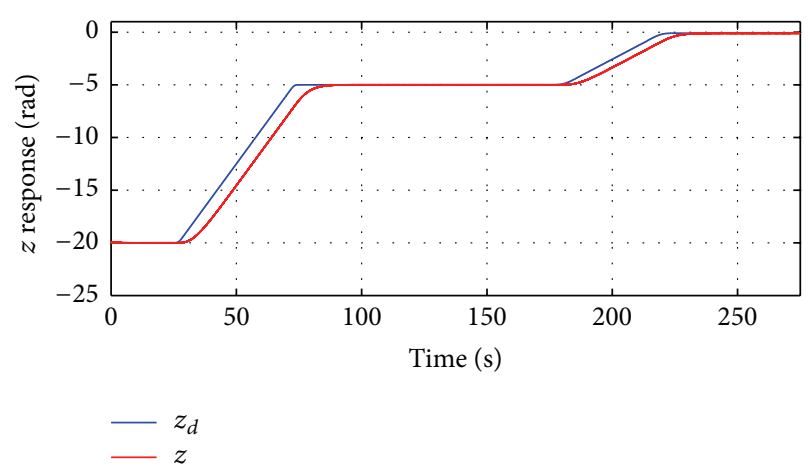

FIGURE 19: Emersion maneuver reference $z_{d}(t)$ and time response $z(t)$.

$$
\begin{array}{r}
\text { from } \mathbf{q}_{d 1}=\left(\begin{array}{c}
0 \\
-0.05 \\
-20
\end{array}\right) \text { to } \mathbf{q}_{d 2}=\left(\begin{array}{c}
\frac{\pi}{3} \\
-0.05 \\
-5
\end{array}\right) \\
t_{1}=25 \text { to } t_{2}=75 \mathrm{~s}, \\
\text { from } \mathbf{q}_{d 2}=\left(\begin{array}{c}
\frac{\pi}{3} \\
-0.05 \\
-5
\end{array}\right) \text { to } \mathbf{q}_{d 3}=\left(\begin{array}{c}
\frac{\pi}{3} \\
-0.05 \\
-5
\end{array}\right) \\
t_{2}=75 \text { to } t_{3}=175 \mathrm{~s},
\end{array}
$$




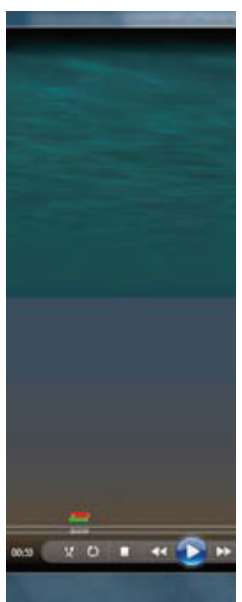

$t=25 \mathrm{~s}$

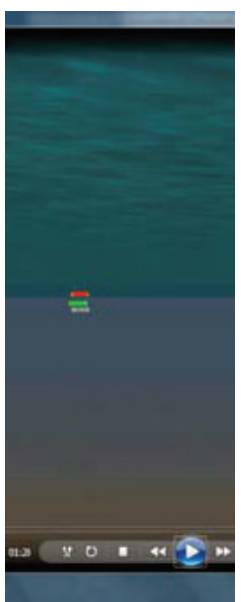

$t=50 \mathrm{~s}$

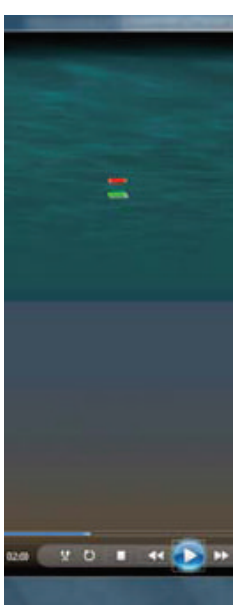

$t=75 \mathrm{~s}$

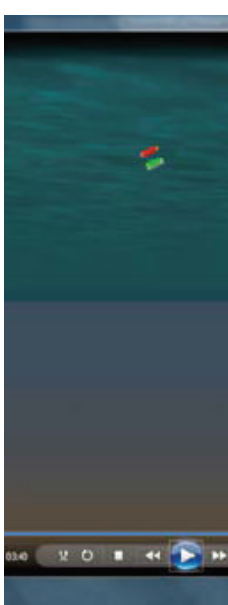

$t=190 \mathrm{~s}$

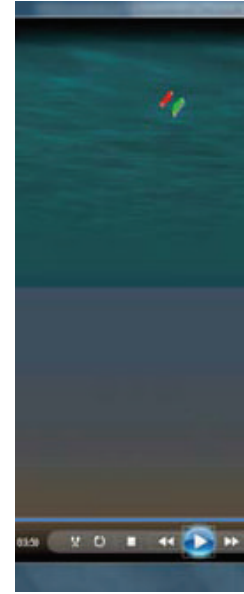

$t=210 \mathrm{~s}$

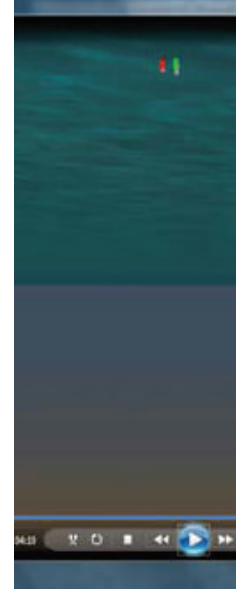

$t=225 \mathrm{~s}$

Figure 20: Graphical view of the closed loop simulation with Matlab-OrcaFlex.

$$
\text { from } \begin{aligned}
\mathbf{q}_{d 3}= & \left(\begin{array}{c}
\frac{\pi}{3} \\
-0.05 \\
-5
\end{array}\right) \text { to } \mathbf{q}_{d 4}=\left(\begin{array}{c}
\frac{\pi}{3} \\
-0.05-\frac{\pi}{2} \\
-0.1
\end{array}\right) \\
t_{3} & =175 \text { to } t_{4}=225 \mathrm{~s},
\end{aligned}
$$$$
\begin{aligned}
\text { from } \mathbf{q}_{d 4} & =\left(\begin{array}{c}
\frac{\pi}{3} \\
-0.05-\frac{\pi}{2} \\
-0.1
\end{array}\right) \text { to } \\
\mathbf{q}_{d f} & =\left(\begin{array}{c}
\frac{\pi}{3} \\
-0.05-\frac{\pi}{2} \\
-0.1
\end{array}\right)
\end{aligned}
$$$$
t_{4}=225 \text { to } t_{f}=275 \mathrm{~s} \text {. }
$$

Figures 17-19 illustrate the excellent response of the system during a complete emersion sequence. It will be observed that the initial posture $\mathbf{q}_{d 0}$ is equivalent to that shown in Figure 2, while the final posture $\mathbf{q}_{d f}$ is equivalent to that shown in Figure 3 (right), corresponding with the normal submerged operation posture for energy harnessing and the normal floating posture for maintenance tasks. Very similar results were obtained by integrating the dynamics of the laboratory prototype into the simulation environment Orcina OrcaFlex [43]. OrcaFlex is one of the world's leading software packages for the design and analysis of a wide range of marine systems (riser systems, mooring systems, installation planning, towed systems, marine renewables, etc.) and is considered validated and certified software for anchor marine systems. The control signals given in (33) were computed in the runtime of Matlab which is connected to OrcaFlex through an external DLL function which is responsible for obtaining the magnitudes from the simulated responses and providing the control signals from and to OrcaFlex with a time sampling of $10 \mathrm{~ms}$. Figure 20 displays a visual sequence of the response of the 3 -DoF prototype when following the references given in (39).

\section{Conclusions}

This paper proposes a new dynamics model that can be used to control a family of submarine electrical generators that was conceived to harness energy from marine currents. The submarine generator is provided with three degrees of freedom by which it is possible to perform closed loop emersion and immersion maneuvers. The dynamic model proposed is based solely on the definition of four lumped masses placed on a plane and a matrix that permits the forces from the three buoyancy actuators to be converted into two torques and a vertical force that are responsible for the rotations and the vertical displacement of the whole system. The model developed exhibits a nonlinear, strongly coupled, and timevarying behavior between buoyancy forces produced by the three actuators and the posture magnitudes measured using a depth sensor and a three-dimensional inclinometer on which the angle rotation around the $z$-axis is ignored. As a first conclusion, we should note that the dynamic model developed in this research is sufficiently precise to describe the underwater 3-DoF tidal energy converter motions and sufficiently simple to be used in the control law design.

The proposed control scheme with which to control the generator using only depth and orientation measurements is, meanwhile, based on three stages: (a) the definition of an uncoupling matrix is used to decouple the generator motions for nonsingular values of orientation; this matrix can be easily computed in real-time and allows the generator to be handled in an uncoupled manner, even in open loop; (b) it is a nonlinear uncoupling-based model for the compensation of the centrifugal and Coriolis torques; and (c) it is a proportionalderivative (PD) control action. The closed loop dynamics was chosen by simply designing proportional and derivative 
matrix gains. The proposed control allows the generator to perform fully automatic closed loop emersion/immersion maneuvers from a submerged position with vertical orientation to a floating posture with a horizontal orientation by using time-varying smooth reference trajectories. A last obtained conclusion is that the control law implemented in this work demonstrates that it is a simple control strategy, which is computationally efficient and easily implementable in a microprocessor-/microcontroller-based system.

Finally, based on more than satisfactory numerical simulations achieved in this research, various experimental branches are now the focus of our attention and are detailed as follows. Our intention is (a) to finalize the construction of a real small-sized laboratory prototype; (b) to use realtime experiments to validate the proposed research; and (c) to study different control strategies and new trajectory generations in order to improve the quality of the closed loop emersion/immersion maneuvers. These will be the main topics of our future research.

\section{Appendices}

\section{A. Coefficients of the Inertia Matrix $\mathrm{J}_{\mathrm{LOC}}$}

The local positions of each of the three masses located at their respective center of gravity on each torpedo and which are taken from (6) are used to obtain the equivalent inertia matrix $\mathbf{J}_{\text {LOC }}$ with regard to the local reference system. It will be noted that the central mass $m_{\mathrm{CG}}$ does not produce any inertia value in accordance with its local position given by (5)

$$
\begin{aligned}
& I_{x x}=\sum_{i=1}^{3} m_{i} \cdot\left(y_{i}^{2}+z_{i}^{2}\right)=\left(m_{1}+m_{2}+m_{3}\right) \cdot L^{2}, \\
& I_{y y}=\sum_{i=1}^{3} m_{i} \cdot\left(x_{i}^{2}+z_{i}^{2}\right)=\left(\frac{m_{1}+m_{2}}{4}+m_{3}\right) \cdot L^{2}, \\
& I_{z z}=\sum_{i=1}^{3} m_{i} \cdot\left(x_{i}^{2}+y_{i}^{2}\right)=\left(3 \frac{m_{1}+m_{2}}{4}\right) \cdot L^{2}, \\
& P_{x y}=P_{y x}=\sum_{i=1}^{3} m_{i} \cdot\left(x_{i} \cdot y_{i}\right)=0, \\
& P_{x z}=P_{z x}=\sum_{i=1}^{3} m_{i} \cdot\left(x_{i} \cdot z_{i}\right)=0, \\
& P_{y z}=P_{z y}=\sum_{i=1}^{3} m_{i} \cdot\left(y_{i} \cdot z_{i}\right)=\left(\sqrt{3} \frac{m_{2}-m_{1}}{4}\right) \cdot L^{2} .
\end{aligned}
$$

Matrix $\mathbf{J}_{\text {LOC }}$ in (17) is then obtained from (16) using the coefficients obtained above from (A.1).

\section{B. Obtaining Matrices $M\left(q_{R}\right)$ and $C\left(q, \dot{q}_{R}\right)$}

Equation (23) was obtained from (19)-(22). This appendix shows how the coefficients of matrices $\mathbf{M}\left(\mathbf{q}_{\mathbf{R}}\right)$ and $\mathbf{C}\left(\mathbf{q}_{\mathbf{R}}, \dot{\mathbf{q}}_{\mathbf{R}}\right)$ were obtained. Firstly, partial derivatives with regard to angular velocities were obtained. Note that the $\Omega_{x}$ and $\Omega_{y}$ notations are maintained as angular velocities in these first equations

$$
\begin{aligned}
& \frac{\partial \mathbf{L}}{\partial \dot{\varphi}_{x}}=\Omega_{x}\left(I_{x x} c^{2} \theta_{y}+I_{z z} s^{2} \theta_{y}\right) \\
& \quad+\Omega_{y}\left[\left(I_{x x}-I_{z z}\right) s \theta_{y} c \theta_{y} s \varphi_{x}-P_{y z} s \theta_{y} c \varphi_{x}\right] \\
& \frac{\partial \mathbf{L}}{\partial \dot{\theta}_{y}}=\Omega_{x}\left[\left(I_{x x}-I_{z z}\right) s \theta_{y} c \theta_{y} s \varphi_{x}-P_{y z} s \theta_{y} c \varphi_{x}\right] \\
& \quad+\Omega_{y}\left[\left(I_{x x} s^{2} \theta_{y}+I_{z z} c^{2} \theta_{y} s^{2}\right) \varphi_{x}+I_{y y} c^{2} \varphi_{x}\right. \\
& \left.\quad+2 P_{y z} c \theta_{y} s \varphi_{x} c \varphi_{x}\right] .
\end{aligned}
$$

Partial derivatives with regard to generalized coordinated rotation are then obtained:

$$
\begin{aligned}
& \frac{\partial \mathbf{L}}{\partial \varphi_{x}}=\Omega_{x} \Omega_{y}\left[\left(I_{x x}-I_{z z}\right) s \theta_{y} c \theta_{y} c \varphi_{x}+P_{y z} s \theta_{y} s \varphi_{x}\right] \\
& \quad+\Omega_{y}^{2}\left[\left(I_{x x} s^{2} \theta_{y}-I_{y y}+I_{z z} c^{2} \theta_{y}\right) s \varphi_{x} c \varphi_{x}\right. \\
& \left.\quad+P_{y z}\left(c^{2} \varphi_{x}-s^{2} \varphi_{x}\right)\right] \\
& \frac{\partial \mathbf{L}}{\partial \theta_{y}}=\Omega_{x}^{2}\left(-I_{x x}+I_{z z}\right) s \theta_{y} c \theta_{y} \\
& \quad+\Omega_{x} \Omega_{y}\left[\left(I_{x x}-I_{z z}\right)\left(c^{2} \theta_{y}-s^{2} \theta_{y}\right) s \varphi_{x}\right. \\
& \left.\quad-P_{y z} c \theta_{y} c \varphi_{x}\right]+\Omega_{y}^{2}\left[\left(I_{x x}-I_{z z}\right) s \theta_{y} c \theta_{y} s^{2} \varphi_{x}\right. \\
& \left.\quad-P_{y z} s \theta_{y} s \varphi_{x} c \varphi_{x}\right] .
\end{aligned}
$$

From here on, in order to obtain time derivatives of $\partial \mathbf{L} / \partial \dot{\varphi}_{x}$ and $\partial \mathbf{L} / \partial \dot{\theta}_{y}$, angular velocities $\Omega_{x}$ and $\Omega_{y}$ are substituted for their respective angular rotation time derivatives

$$
\begin{aligned}
& \Omega=\left(\begin{array}{c}
\Omega_{x} \\
\Omega_{y} \\
\Omega_{z}
\end{array}\right)=\left(\begin{array}{c}
\dot{\varphi}_{x} \\
\dot{\theta}_{y} \\
0
\end{array}\right), \\
& \frac{d}{d t}\left(\frac{\partial \mathbf{L}}{\partial \dot{\varphi}_{x}}\right)=\ddot{\varphi}_{x}\left(I_{x x} c^{2} \theta_{y}+I_{z z} s^{2} \theta_{y}\right) \\
&+\ddot{\theta}_{y}\left(\left(I_{x x}-I_{z z}\right) s \theta_{y} c \theta_{y} s \varphi_{x}-P_{y z} s \theta_{y} c \varphi_{x}\right. \\
&\left.-I_{z z} c \theta_{y} s \theta_{y} s \varphi_{x}\right)+\dot{\varphi}_{x} \dot{\theta}_{y}\left(-2 I_{x x} c \theta_{y} s \theta_{y}\right. \\
&\left.+2 I_{z z} s \theta_{y} c \theta_{y}\right)+\dot{\theta}_{y}^{2}\left[\left(I_{x x}-I_{z z}\right)\left(c^{2} \theta_{y}-s^{2} \theta_{y}\right) s \varphi_{x}\right. \\
&\left.-P_{y z} c \theta_{y} c \varphi_{x}\right]+\dot{\theta}_{y} \dot{\varphi}_{x}\left[\left(I_{x x}-I_{z z}\right) s \theta_{y} c \theta_{y} c \varphi_{x}\right. \\
&\left.+P_{y z} s \theta_{y} s \varphi_{x}\right],
\end{aligned}
$$




$$
\begin{aligned}
& \frac{d}{d t}\left(\frac{\partial \mathbf{L}}{\partial \dot{\theta}_{y}}\right)=\ddot{\varphi}_{x}\left[\left(I_{x x}-I_{z z}\right) s \theta_{y} c \theta_{y} s \varphi_{x}\right. \\
& \left.\quad-P_{y z} s \theta_{y} c \varphi_{x}\right]+\ddot{\theta}_{y}\left[\left(I_{x x} s^{2} \theta_{y}+I_{z z} c^{2} \theta_{y}\right) s^{2} \varphi_{x}\right. \\
& \left.\quad+I_{y y} c^{2} \varphi_{x}+2 P_{y z} c \theta_{y} s \varphi_{x} c \varphi_{x}\right] \\
& \quad+\dot{\varphi}_{x} \dot{\theta}_{y}\left[\left(I_{x x}-I_{z z}\right)\left(c^{2} \theta_{y}-s^{2} \theta_{y}\right) s \varphi_{x}\right. \\
& \left.\quad-P_{y z} c \theta_{y} c \varphi_{x}\right]+\dot{\varphi}_{x}^{2}\left[\left(I_{x x}-I_{z z}\right) s \theta_{y} c \theta_{y} c \varphi_{x}\right. \\
& \left.\quad+P_{y z} s \theta_{y} s \varphi_{x}\right] 2 \dot{\theta}_{y}^{2}\left[\left(I_{x x}-I_{z z}\right) s \theta_{y} c \theta_{y} s^{2} \varphi_{x}\right. \\
& \left.\quad-P_{y z} s \theta_{y} s \varphi_{x} c \varphi_{x}\right] \\
& \quad+2 \dot{\theta}_{y} \dot{\varphi}_{x}\left[\left(I_{x x} s^{2} \theta_{y}+I_{z z} c^{2} \theta_{y}\right) s \varphi_{x} c \varphi_{x}\right. \\
& \left.\quad-I_{y y} s \varphi_{x} c \varphi_{x}+P_{y z}\left(c^{2} \varphi_{x}-s^{2} \varphi_{x}\right) c \theta_{y}\right] .
\end{aligned}
$$

Finally, after rearranging (B.2) to (B.3), one obtains the coefficients of the left hand of (23) which is now reproduced again:

$$
\begin{aligned}
& \mathbf{M}_{\mathbf{R}}\left(\mathbf{q}_{\mathbf{R}}\right) \cdot \ddot{\mathbf{q}}_{\mathbf{R}}+\mathbf{C}_{\mathbf{R}}\left(\mathbf{q}_{\mathbf{R}}, \dot{\mathbf{q}}_{\mathbf{R}}\right) \text {, with } \mathbf{q}_{\mathbf{R}}=\left(\begin{array}{c}
\varphi_{x} \\
\theta_{y}
\end{array}\right), \\
& M_{\mathbf{R}}(1,1)=I_{x x} c^{2} \theta_{y}+I_{z z} s^{2} \theta_{y}, \\
& M_{\mathbf{R}}(1,2)=\left(I_{x x}-I_{z z}\right) s \theta_{y} c \theta_{y} s \varphi_{x}-P_{y z} s \theta_{y} c \varphi_{x}, \\
& M_{\mathbf{R}}(2,1)=\left(I_{x x}-I_{z z}\right) s \theta_{y} c \theta_{y} s \varphi_{x}-P_{y z} s \theta_{y} c \varphi_{x}, \\
& M_{\mathbf{R}}(2,2)=\left(I_{x x} s^{2} \theta_{y}+I_{z z} c^{2} \theta_{y}\right) s^{2} \varphi_{x}+I_{y y} c^{2} \varphi_{x} \\
& \quad+2 P_{y z} c \theta_{y} s \varphi_{x} c \varphi_{x}, \\
& C_{\mathbf{R}}(1)=2 \dot{\varphi}_{x} \dot{\theta}_{y}\left[\left(I_{z z}-I_{x x}\right) c \theta_{y} s \theta_{y}\right] \\
& \quad+\dot{\theta}_{y}^{2}\left\{I_{x x}\left[c^{2} \theta_{y}-s^{2} \theta_{y}\left(1+c \varphi_{x}\right)\right]\right. \\
& \left.\quad-I_{z z}\left[c^{2} \theta_{y}\left(1+c \varphi_{x}\right)-s^{2} \theta_{y}\right]\right\} \\
& \quad+\dot{\theta}_{y}^{2}\left\{-P_{y z} c \theta_{y}\left[c \varphi_{x}\left(1+c \varphi_{x}\right)-s^{2} \varphi_{x}\right]\right. \\
& \left.\quad+I_{y y} s \varphi_{x} c \varphi_{x}\right\}, \\
& \quad+2 \dot{\varphi}_{x} \dot{\theta}_{y}\left[\left(I_{x x} s^{2} \theta_{y}+I_{z z} c \theta_{y}{ }^{2} \theta_{y}\right) s \varphi_{x} c \varphi_{x} c \varphi_{x}\right] \\
& C_{\mathbf{R}}(2)=\dot{\varphi}_{x}^{2}\left[\left(I_{x x}-I_{z z}\right) s \theta_{y} c \theta_{y}\left(1+c \varphi_{x}\right)\right] \\
& \quad\left(\theta_{y} c \theta_{y} s^{2} \varphi_{x}-P_{y z} s \theta_{y} s \varphi_{x} c \varphi_{x}\right] .
\end{aligned}
$$

\section{Conflict of Interests}

The authors declare that there is no conflict of interests regarding the publication of this paper.

\section{Acknowledgment}

This work has been supported by Spanish Ministerio de Economía y Competitividad under Research Grants DPI201124113 and DPI2014-53499R.

\section{References}

[1] A. Brito and J. L. Villate, Eds., Annual Report. Implementing Agreement on Ocean Energy Systems-2013, The Executive Committee of Ocean Energy Systems, 2014.

[2] J. Hardisty, The Analysis of Tidal Stream Power, Wiley, 2009.

[3] J. King and T. Tryfonas, "Tidal stream power technology- state of the art," in Proceedings of the IEEE (OCEANS '09), pp. 1-8, Bremen, Germany, May 2009.

[4] M. J. Khan, G. Bhuyan, M. T. Iqbal, and J. E. Quaicoe, "Hydrokinetic energy conversion systems and assessment of horizontal and vertical axis turbines for river and tidal applications: a technology status review," Applied Energy, vol. 86, no. 10, pp. $1823-1835,2009$.

[5] C. M. Johnstone, D. Pratt, J. A. Clarke, and A. D. Grant, "A techno-economic analysis of tidal energy technology," Renewable Energy, vol. 49, pp. 101-106, 2013.

[6] A. S. Bahaj, "Generating electricity from the oceans," Renewable and Sustainable Energy Reviews, vol. 15, no. 7, pp. 3399-3416, 2011.

[7] F. O. Rourke, F. Boyle, and A. Reynolds, "Tidal energy update 2009," Applied Energy, vol. 87, no. 2, pp. 398-409, 2010.

[8] A. López, J. A. Somolinos, and L. R. Núñez, "Energetic modelling of primary converters for marine renewable energies," Revista Iberoamericana de Automatica e Informatica Industrial, vol. 11, no. 2, pp. 224-235, 2014.

[9] Mustang Engineering. Offshore Magazine, "Deepwater solutions \& records for concept selection," 2014, http://www.offshore-mag .com/maps-posters.html.

[10] M. Shafiee, "Maintenance logistics organization for offshore wind energy: current progress and future perspectives," Renewable Energy, vol. 77, pp. 182-193, 2015.

[11] A. F. O. Falcão, J. C. C. Henriques, and J. J. Cândido, "Dynamics and optimization of the OWC spar buoy wave energy converter," Renewable Energy, vol. 48, pp. 369-381, 2012.

[12] M. S. Güney and K. Kaygusuz, "Hydrokinetic energy conversion systems: a technology status review," Renewable and Sustainable Energy Reviews, vol. 14, no. 9, pp. 2996-3004, 2010.

[13] S. E. B. Elghali, R. Balme, K. Le Saux, M. E. H. Benbouzid, J. F. Charpentier, and F. Hauville, "A simulation model for the evaluation of the electrical power potential harnessed by a marine current turbine," IEEE Journal of Oceanic Engineering, vol. 32, no. 4, pp. 786-797, 2007.

[14] S. E. Ben Elghali, M. E. H. Benbouzid, and J. F. Charpentier, "Marine tidal current electric power generation technology: state of the art and current status," in Proceedings of the IEEE International Electric Machines and Drives Conference (IEMDC '07), pp. 1407-1412, May 2007.

[15] H. H. Aly, "The current status of wind and tidal in-stream electric energy resources," American Journal of Electrical Power and Energy Systems, vol. 2, no. 2, pp. 23-40, 2013.

[16] A. López, J. A. Somolinos, L. R. Núñez, and J. Valle, "Dynamic behavior of a second generation hydrokinetic converter," in Proceedings of the IEEE International Conference on Oceanic Engineering (OCEANS '11), Santander, Spain, 2011. 
[17] 2015, http://www.seageneration.co.uk/.

[18] Consulted May, 2015, http://wavestarenergy.com/.

[19] 2015, http://atlantisresourcesltd.com/.

[20] 2015, http://www.hammerfeststrom.com/.

[21] May 2015, http://www.openhydro.com/home.html.

[22] 2015, http://www.tocardo.com/.

[23] May 2015, http://www.verdantpower.com/.

[24] 2015, http://www.alstom.com/products-services/product-catalogue/power-generation/renewable-energy/ocean-energy/tidalenergy/tidal-power/.

[25] May 2015, http://www.nautricity.com/cormat/.

[26] 2015, http://www.pulsetidal.com/.

[27] Spanish Patent and Trademark Office, "Patent number concession n P200700987," UPM. OEPM, Madrid, Spain, 2008.

[28] IEC-TC214, Tecnhical Specification: Marine Energy. Wave, Tidal and Other Water Current Converters. Part 1: Terminology, IEC/TS 62600-1, International Electrotechnic Commision, Ginebra, Switzerland, 2011.

[29] A. J. Soressen, "Marine control systems," Tech. Rep. UK-13-76, Norwegian University of Science and Technology, 2013.

[30] T. I. Fossen, Handbook of Marine Craft Hydrodynamics and Motion Control, Wiley, Chichester, UK, 2011.

[31] J. A. Somolinos, V. Feliu, and L. Sánchez, "Design, dynamic modelling and experimental validation of a new three-degreeof-freedom flexible arm," Mechatronics, vol. 12, no. 7, pp. 919948, 2002.

[32] V. Feliu, J. A. Somolinos, and A. García, "Inverse dynamics based control system for a three-degree-of-freedom flexible arm," IEEE Transactions on Robotics and Automation, vol. 19, no. 6, pp. 1007-1014, 2003.

[33] R. Morales, J. A. Somolinos, and J. A. Cerrada, "Dynamic model of a stair-climbing mobility system and its experimental validation," Multibody System Dynamics, vol. 28, no. 4, pp. 349367, 2012.

[34] R. Morales, J. A. Somolinos, and J. A. Cerrada, "Dynamic control of a reconfigurable stair-climbing mobility system," Robotica, vol. 31, no. 2, pp. 295-310, 2013.

[35] F. M. White, Fluid Mechanics, McGraw-Hill, New York, NY, USA, 4th edition, 1998.

[36] B. R. Munson, D. F. Young, and T. H. Okiishi, Fundamentals of Fluid Mechanics-International Student Version, Wiley, New York, NY, USA, 6th edition, 1990.

[37] S. L. Dixon and C. A. Hall, Fluid Mechanics and Thermodynamics of Turbomachinery, Butterworth-Heinemann, Elsevier, 7th edition, 2013.

[38] F. H. Imlay, "The complete expressions for added mass of a rigid body moving in an ideal fluid," David Taylor Model Basin Report 152B, Department of the Navy, 1961.

[39] A. López, L. R. Núñez, J. Somolinos, E. Novoa, and A. Carneros, "Methodology and results of the sea trials for a second generation tidal converter," in Maritime Technology and Engineering, pp. 1213-1221, Taylor \& Francis Group, London, UK, 2015.

[40] V. Feliu, K. S. Rattan, and H. B. Brown, "Control of flexible arms with friction in the joints," IEEE Transactions on Robotics and Automation, vol. 9, no. 4, pp. 467-475, 1993.

[41] M. Hashimoto, F. Oba, and H. Nakahara, "Trajectory generation and tracking control methods for a multiple transfer robots system," in Proceedings of the IEEE/RSJ International Workshop on Intelligent Robots and Systems (IROS '91), pp. 799-804, 1991.
[42] S. Saravana Perumaal and N. Jawahar, "Automated trajectory planner of industrial robot for pick-and-place task," International Journal of Advanced Robotic Systems, vol. 10, p. 100, 2013.

[43] 2015, http://www.orcina.com/. 


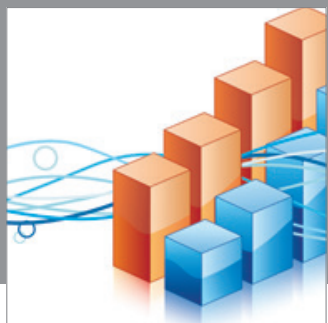

Advances in

Operations Research

mansans

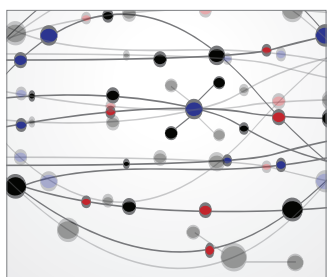

The Scientific World Journal
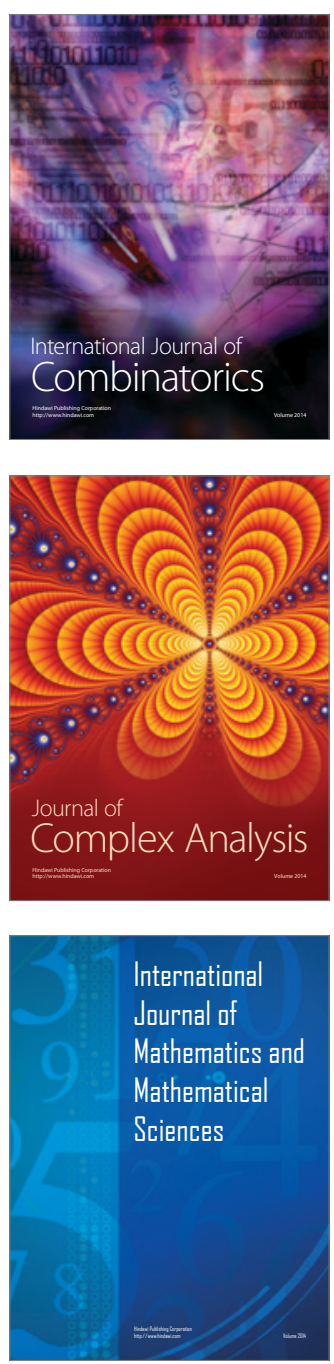
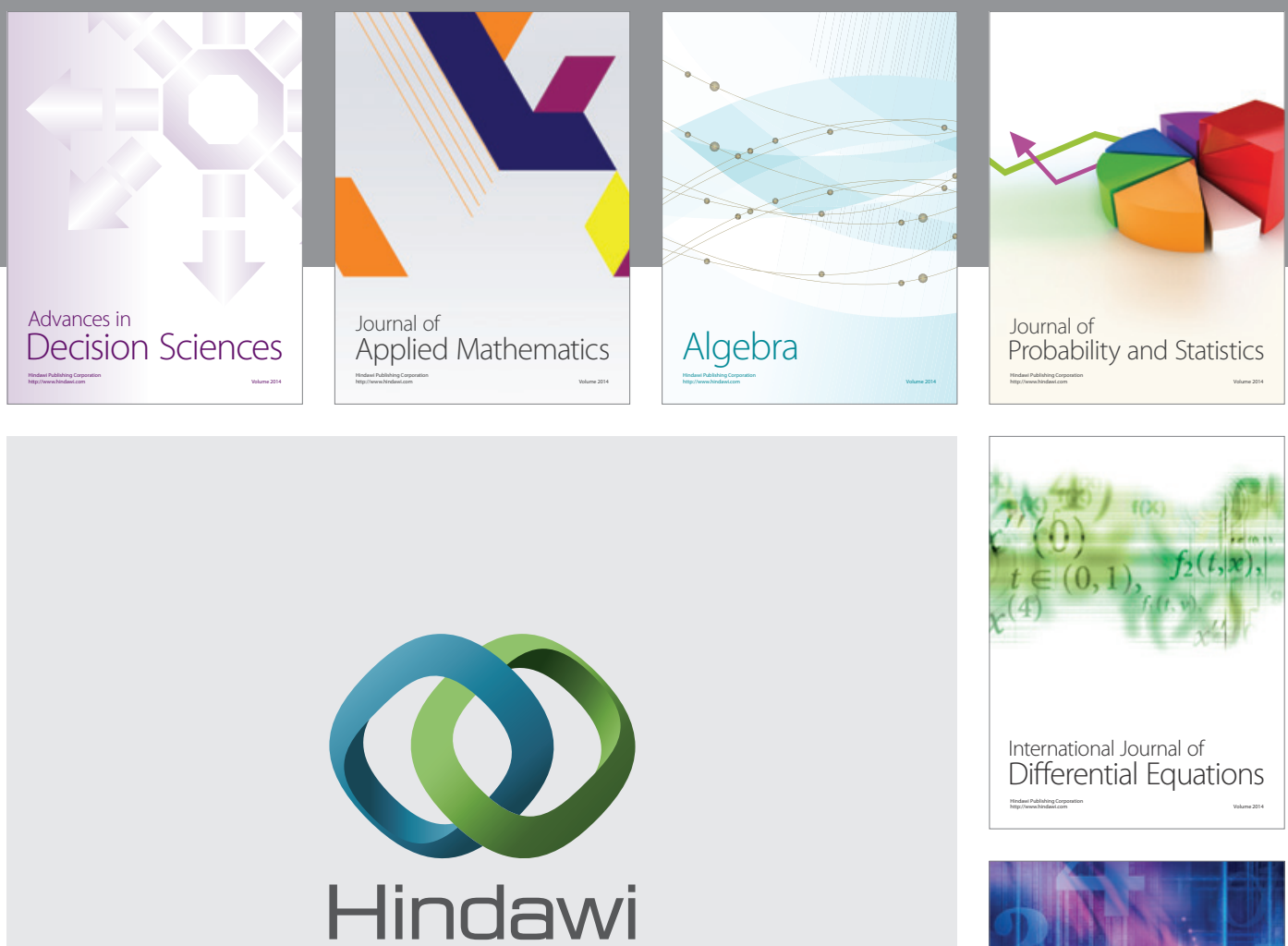

Submit your manuscripts at http://www.hindawi.com
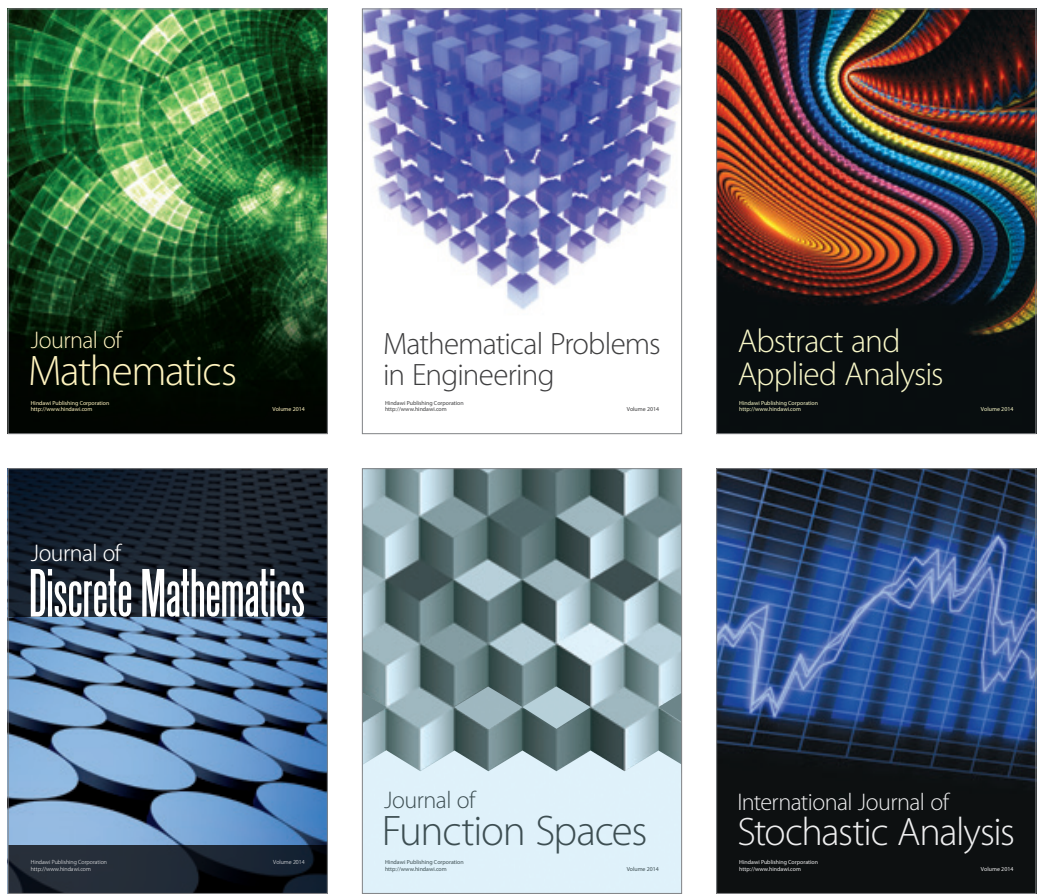

Journal of

Function Spaces

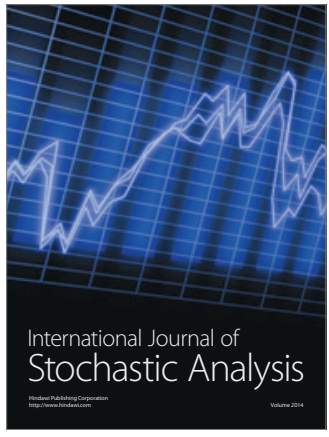

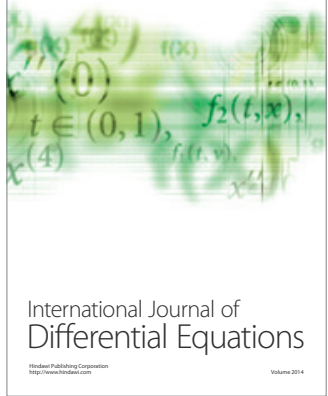
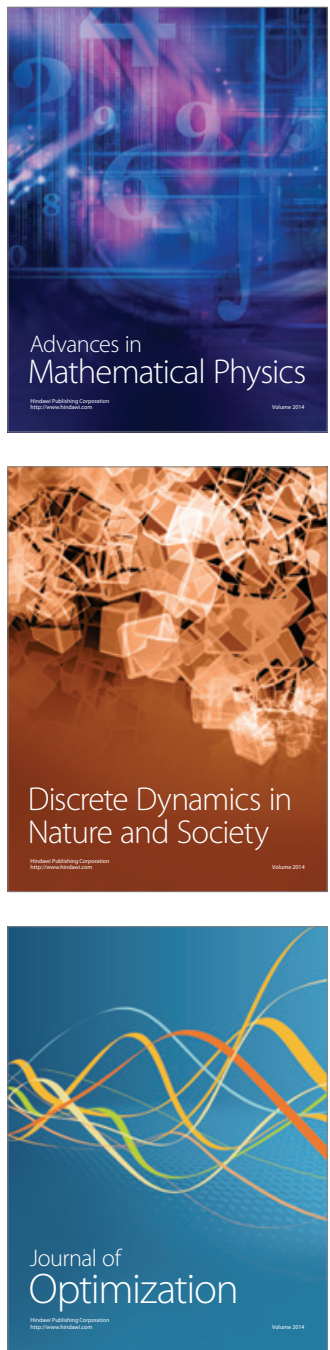